\title{
Smooth second-order sliding mode controller for multivariable mechanical systems
}

\author{
Panitnart Chawengkrittayanont ${ }^{1} \cdot$ Chutiphon Pukdeboon $^{1} \cdot$ Suwat Kuntanapreeda ${ }^{2} \cdot$ Elvin J. Moore ${ }^{3,4}($ C)
}

Received: 26 May 2021 / Accepted: 9 September 2021

Published online: 27 September 2021

(c) The Author(s) 2021 OPEN

\begin{abstract}
This paper proposes a smooth second-order sliding mode controller for a class of multi-input multi-output mechanical systems with uncertain parameters and external disturbances. Since the control law is smooth, the chattering effect that can occur with non-smooth controllers is reduced. Lyapunov-based theorems are used to prove global and finite-time convergence of the sliding mode controller. Numerical simulations are presented to illustrate the performance of the proposed controller by applying it first to a variable-length pendulum and then to a two-link robotic manipulator. For the robotic manipulator, a detailed comparison is given of the finite-time convergence and chattering properties of the proposed controller, a super-twisting controller and a super-twisting like controller.
\end{abstract}

\section{Article Highlights}

- A new smooth second-order sliding mode controller is proposed for multivariablesystems with uncertain parameters and external disturbances.

- Global finite-time convergence of the controller is proved using Lyapunov theory.

- Numerical simulations are used to show the performance of the controller on thevariable-length pendulum and two-link robotic manipulator.

Keywords Finite-time convergence $\cdot$ Second-order sliding mode control $\cdot$ Smooth sliding mode control $\cdot$ Multi-input multi-output system

Mathematics Subject Classification 93D40 $\cdot 93 \mathrm{C} 10 \cdot 93 \mathrm{C} 15 \cdot 93 \mathrm{C} 85 \cdot 70 \mathrm{Q} 05$

\section{Introduction}

Modern mechanical systems (e.g., manipulators, flexible structures, autonomous aerial vehicles, spacecraft, etc.) require high performance control schemes. These systems usually contain uncertain parameters and are always subject to external disturbances. Thus, control schemes that make systems insensitive to uncertain parameters and external disturbances are highly desirable. Among the existing control methods, sliding mode control (SMC), originally proposed by Utkin [1], has been considered as one of the best methods because of its simplicity and high

$\triangle$ Elvin J. Moore, elvin.j@sci.kmutnb.ac.th | 'Department of Mathematics, Faculty of Applied Science, King Mongkut's University of Technology North Bangkok, Bangkok, Thailand. ${ }^{2}$ Department of Mechanical and Aerospace Engineering, Faculty of Engineering, King Mongkut's University of Technology North Bangkok, Bangkok, Thailand. ${ }^{3}$ Department of Mathematics, Faculty of Applied Science, King Mongkut's University of Technology North Bangkok, Bangkok, Thailand. ${ }^{4}$ Centre of Excellence in Mathematics, CHE, Si Ayutthaya Road, Bangkok 10400, Thailand. 
robustness. SMC has been successfully applied to many types of dynamical systems including discrete-time systems [2, 3], multi-input multi-output (MIMO) systems [4, 5], time-delay systems [6], large-scale and infinite-dimensional systems $[7,8]$, and stochastic systems [9-11].

Using the SMC, a suitable sliding surface is first defined and then a controller based on the signum function is designed to drive the system state to the defined sliding surface (see, e.g., $[12,13])$. However, the SMC can produce high-frequency amplitude oscillations (chattering) due to the discontinuous control signal. For mechanical systems, this chattering can cause vibration-induced fatigue. It also decreases the control performance and may cause instability of the control system. Thus, chattering should be avoided or reduced to a low level.

Higher-order sliding mode control (HOSMC) was introduced in $[14,15]$ in an attempt to reduce the chattering problem while still maintaining the advantages of the conventional SMC. Twisting and super-twisting algorithms are two of the best-known HOSMC methods $[16,17]$. In HOSMC, the signum function acts on higher-order timederivatives of sliding variables, instead of only on the first time-derivative as in conventional SMC controllers. The HOSMC has also been claimed to provide better accuracy with respect to discrete sampling times. Recent detailed discussions and comparisons of HOSMC and conventional SMC have been given by Shtessel et al. [18] and Utkin and colleagues [19-21].

A number of different algorithms based on HOSMC have been developed to achieve finite-time stability in a variety of systems. An HOSMC approach for stabilizing nonholonomic perturbed systems has been presented in [22]. The results showed that the trajectory of the system converged in finite time. In [23], an optimal-control based HOSMC approach with finite-time convergence was proposed, and the approach was successfully applied to control electropneumatic actuators. In [24], a HOSMC scheme for uncertain nonlinear systems was proposed. By utilizing an integral sliding surface, robustness of the control scheme was ensured. However, this method has the main disadvantage that it depends on the initial condition of the system which usually cannot be known precisely. A geometric homogeneity based HOSMC was proposed in [25] for a chain of integrator systems. The controller yielded finite-time stability for the control system. A novel geometric homogeneity-based HOSMC was developed in [26] and successfully applied to a MIMO nonlinear system. A chattering-free terminal sliding mode control of an $n$ thorder system was developed in [27]. This controller drives the system state to zero in finite time.

A smooth modified super twisting (MST) sliding mode scheme has been developed from a smooth second-order sliding mode control [28] by [29] by adding linear correction terms to improve the performance of the closed-loop system. The smooth MST was proved to have finite-time convergence by using strong Lyapunov design.

There have been a number of papers discussing applications of HOSMC to mechanical systems. For example, Chutiphon et al. [30] designed an HOSM controller for attitude tracking control of a spacecraft. Mondal and Mahanta [31] developed an adaptive second-order sliding mode controller for robotic manipulators. Guendouzi et al. [32] applied an HOSMC to a small-size autonomous helicopter. Finite-time controllers that guarantee finite-time convergence of the system states to a desired state have also been discussed recently in [33-36].

There have also been applications of smooth second-order sliding mode control (SOSMC) to a variety of mechanical systems. For example, Shtessel et al. [28] proposed a smooth SOSMC for a target performing evasive maneuvers to escape an interceptor missile and they proved the finite-time convergence of the method using a homogeneity based technique. Wang [37] proposed an adaptive smooth SOSMC and showed its application to missile guidance. Wang proved finite-time convergence of the adaptive smooth SOSMC by using a quadratic Lyapunov function. Yang et al. [38] proposed a fast smooth SOSMC for a class of stochastic systems with colored noise and proved finite-time convergence of the controller for a second-order nonlinear stochastic system by using stochastic Lyapunov techniques.

In this paper, we propose a new smooth SOSMC controller with the finite-time convergence property for multivariable mechanical systems in the presence of uncertain parameters and external disturbances. The controller is a smooth continuous controller with reduced chattering. The control law generalizes a sliding surface developed in [25], which is based on geometric homogeneity. The novelties of our work are as follows:

1. Standard SOSMC methods can include discontinuous first-order time derivatives of the sliding variables which can induce chattering in the controller. Our proposed smooth SOSMC includes continuous first-order time derivatives of the sliding variables and hence chattering is reduced.

2. The proposed multivariable smooth SOSMC algorithms are analyzed by using the Lyapunov-based ideas from Moreno and Osorio [39]. However, our analysis differs from that of Moreno and Osorio because we use a Lyapunov function containing state variables with an unknown fractional power to prove the finitetime stability of the controller.

The paper is organized as follows. In Sect. 2, basic lemmas and theorems are presented and the details of the class 
of multivariable mechanical systems that we study in this paper are discussed. In Sect. 3, the second-order sliding surface used in this paper is described and the properties of the proposed finite-time smooth SOSMC law are analyzed. A Lypunov-based analysis is given to prove the global stability of the controller and finite-time stability of the system. In Sect. 4, results of numerical simulations are presented showing the finite-time convergence of the proposed controller for a variable-length pendulum [40] and for a two-link robotic manipulator [41]. For the manipulator, a detailed comparison is given of the behavior of the finite-time convergence and chattering properties of the proposed smooth SOSMC and the behavior of a super-twisting (STW) algorithm [16] and a super-twisting like (STL) algorithm [17]. Finally, Sects. 5 and 6 contain discussion and conclusions.

\section{Preliminaries}

\subsection{Lemmas and theorems}

Theorem 1 (Lyapunov Theorem) (see, e.g., [42-44]). Consider the autonomous system

$\dot{x}=f(x)$,

where $f: \mathcal{D} \subset \mathfrak{R}^{n} \rightarrow \mathfrak{R}^{n}$ is continuous and $f(0)=0$. The equilibrium point 0 of system (1) is globally stable for all initial points $x(0) \in \mathcal{D}$ if there exists a continuously differentiable function $V: \mathcal{D} \rightarrow \mathfrak{R}^{n}$, called a Lyapunov function, such that
(a) $\quad V(x)$ is positive definite in $\mathcal{D}$,
(b) $V(x)$ has a unique minimum at the equilibrium point 0 ,
(c) $\quad \dot{V}(x)=\frac{d V(x(t))}{d t}$ is negative semi-definite in $\mathcal{D}$.

If, in addition, $\dot{V}(x)$ is negative definite in $\mathcal{D}$, then the equilibrium point 0 is globally asymptotically stable for all initial points $x(0) \in \mathcal{D}$.

Lemma 1 ([45]) Consider a positive-definite function $V(t)$, which satisfies the following differential inequality

$\dot{V}(t) \leq-\delta V(t)-\beta V(t)^{\eta} \quad \forall t \geq t_{0}, V\left(t_{0}\right) \geq 0$,

where $\delta$ and $\beta$ are two positive coefficients, and $\eta$ is a positive number with $0<\eta<1$. Then, the function $V(t)$ converges to zero in the finite time

$T=t_{0}+\frac{1}{\delta(1-\eta)} \ln \left(\frac{\delta V\left(t_{0}\right)^{1-\eta}+\beta}{\beta}\right)$.

Theorem 2 [25] Consider the system

$$
\begin{gathered}
\dot{x}_{1}=x_{2}, \\
\dot{x}_{2}=x_{3} \\
\vdots \\
\dot{x}_{n}=u,
\end{gathered}
$$

where $u$ is the input of the system given by

$u=-a_{1} \operatorname{sgn}\left(x_{1}\right)\left|x_{1}\right|^{\rho_{1}}-a_{2} \operatorname{sgn}\left(x_{2}\right)\left|x_{2}\right|^{\rho_{2}}-\cdots-a_{n} \operatorname{sgn}\left(x_{n}\right)\left|x_{n}\right|^{\rho_{n}}$

and where sgn $(\cdot)$ is the signum function, the polynomial $\phi(\lambda)=\lambda^{n}+a_{n} \lambda^{n-1}+\ldots+a_{2} \lambda+a_{1}$ is Hurwitz, and $\rho_{1}, \ldots, \rho_{n}$ satisfy $\rho_{i-1}=\frac{\rho_{i} \rho_{i+1}}{2 \rho_{i+1}-\rho_{i}}, i=2, \ldots, n$ with $\rho_{n+1}=1$ and $\rho_{n}=\rho$.

Then, for system (4), there exists a value $\varepsilon \in(0,1)$ such that for every $\rho_{i} \in(1-\varepsilon, 1)$, the origin of system (4) is a globally stable equilibrium in finite time under the input $u$ in (5).

Lemma 2 ([46]) For any $x_{i} \in \mathfrak{R}^{n}, i=1,2, \ldots$, nand $0<\eta \leq 1$, the following inequalities hold

$$
\left(\sum_{i=1}^{n}\left|x_{i}\right|\right)^{\eta} \leq \sum_{i=1}^{n}\left|x_{i}\right|^{\eta} \leq n^{1-\eta}\left(\sum_{i=1}^{n}\left|x_{i}\right|\right)^{\eta} .
$$

\subsection{Mechanical system description}

We consider a class of multivariable mechanical systems given by ([47])

$M(\mathbf{q}) \ddot{\mathbf{q}}+C(\mathbf{q}, \dot{\mathbf{q}}) \dot{\mathbf{q}}+g(\mathbf{q})+D(t, \mathbf{q}, \dot{\mathbf{q}})=\boldsymbol{\tau}$,

where $\mathbf{q} \in \mathfrak{R}^{n}$ is a generalized coordinates vector, $M(\mathbf{q}) \in \mathfrak{R}^{n \times n}$ is an inertia matrix, $C(\mathbf{q}, \dot{\mathbf{q}}) \in \Re^{n \times n}$ is the centrifugal-Coriolis matrix, $g(\mathbf{q}) \in \mathfrak{R}^{n}$ is the gravity force, $D(t, \mathbf{q}, \dot{\mathbf{q}}) \in \Re^{n}$ is a disturbance, and $\tau \in \Re^{n}$ is a control torque. Here, $D(t, \mathbf{q}, \dot{\mathbf{q}})$ can consist of all kinds of disturbance factors, such as parameter uncertainty, unmodeled dynamics, external disturbance, etc. Denoting $\mathbf{v}=\dot{\mathbf{q}} \in \mathfrak{R}^{n}$, the system (7) can be written as

$\dot{\mathbf{q}}=\mathbf{v}, \quad \dot{\mathbf{v}}=\mathbf{u}+\mathbf{f}+\mathbf{d}$,

where $\mathbf{u}=M^{-1} \tau \in \Re^{n}, \mathbf{d}=-M^{-1} D(t, \mathbf{q}, \mathbf{v}) \in \Re^{n}$ and $f=-M^{-1}(C(\mathbf{q}, \mathbf{v}) \mathbf{v}+g(\mathbf{q})) \in \Re^{n}$ can be seen respectively as the control input, the disturbance and the nonlinearity of system (8).

Assumption 1 ([34]) The disturbance vector $\mathbf{d}$ in (8) is a bounded continuous function and its first derivative $\dot{\mathbf{d}}=\bar{\phi}$ is bounded. Thus, there exist positive constants $\overline{\mathrm{d}}$ and $\bar{\phi}$ such that $\|\mathbf{d}\| \leq \overline{\mathbf{d}}$ and $\|\boldsymbol{\phi}\| \leq \bar{\phi}$.

Let the desired position $\mathbf{q}_{d} \in \Re^{n}$ and velocity $\mathbf{v}_{d} \in \Re^{n}$ satisfy the following system 
$\dot{\mathbf{q}}_{d}=\mathbf{v}_{d}, \quad \dot{\mathbf{v}}_{d}=\mathbf{f}_{d}$,

where $\mathbf{f}_{d} \in \Re^{n}$ is a continuous function. We will define $\mathbf{e}=\mathbf{q}-\mathbf{q}_{d} \in \Re^{n}$ and $\varepsilon=\mathbf{v}-\mathbf{v}_{d} \in \Re^{n}$ as tracking errors. From (8) and (9), the error dynamics can be written as

$\dot{\mathbf{e}}=\varepsilon, \quad \dot{\varepsilon}=\mathbf{u}+\mathbf{f}_{e}+\mathbf{d}$,

where $\mathbf{f}_{e}=\mathbf{f}-\mathbf{f}_{d} \in \Re^{n}$, $\mathbf{u}$ is the control and $\mathbf{d}$ is a disturbance.

We introduce the following notation for use in the next section. For a vector $\mathbf{x}=\left[\begin{array}{llll}x_{1} & x_{2} & \ldots & x_{n}\end{array}\right]^{T} \in \mathfrak{R}^{n}$ and $\rho \in \Re$, we define

$\operatorname{sig}(\mathbf{x})^{\rho}=\left[\left|x_{1}\right|^{\rho} \operatorname{sgn}\left(x_{1}\right)\left|x_{2}\right|^{\rho} \operatorname{sgn}\left(x_{2}\right) \ldots\left|x_{n}\right|^{\rho} \operatorname{sgn}\left(x_{n}\right)\right]^{T}$,

where sgn is the signum function.

\section{Main results}

Our objective is to design a smooth SOSMC featuring finite-time convergence to deal with the class of multivariable mechanical systems given by (8). Specifically, a sliding surface and a control law will first be designed so that if the system is on the sliding surface, then the control law will keep the system on the sliding surface and drive the errors $\mathbf{e}$ and $\varepsilon$ in (10) to a neighborhood of zero in finite time. Secondly, a control law is designed to drive the system into a neighborhood of the sliding surface in finite time.

For second-order sliding motion for an $n$-dimensional multi-input multi-output system, it is necessary to specify an equation for the time derivative of the sliding variable $\dot{\mathbf{s}}(t)=\mathbf{0}_{n}$, where $\mathbf{0}_{n}=\left[\begin{array}{llll}0 & 0 & \ldots & 0\end{array}\right]^{T} \in \Re^{n}$ (see, e.g., [28]). The equation for the sliding variable is then obtained as the integral of the $\dot{\mathbf{s}}(t)=\mathbf{0}_{n}$ equation. We propose the following sliding mode equations for a smooth SOSMC that will give finite-time control.

$\mathbf{s}(t)=\varepsilon+\int_{0}^{t}\left(A \operatorname{sig}(\mathbf{e}(\tau))^{\rho_{1}}+B \operatorname{sig}(\varepsilon(\tau))^{\rho_{2}}\right) d \tau=\mathbf{0}_{n^{\prime}}$
$\dot{\mathbf{s}}(t)=\dot{\varepsilon}+A \operatorname{sig}(\mathbf{e}(t))^{\rho_{1}}+B \operatorname{sig}(\varepsilon(t))^{\rho_{2}}=\mathbf{0}_{n^{\prime}}$ for $t \geq 0$,

where $\operatorname{sig}(\mathbf{x})^{\rho}$ is defined in Eq. (11), $A=\operatorname{diag}\left(a_{i}>0\right) \in \mathfrak{R}^{n \times n}, B=\operatorname{diag}\left(b_{i}>0\right) \in \Re^{n \times n} \quad$, $\lambda^{2}+b_{i} \lambda+a_{i}$ is Hurwitz, $0<\rho_{2}<1$ and $\rho_{1}=\frac{\rho_{2}}{2-\rho_{2}}$.

We now define a control vector $\mathbf{u}_{0}$ that will drive the system along the sliding surface to the zero state in finite time. From Eqs. (10) and (12) and setting the disturbance $\mathbf{d}=\mathbf{0}$, the equations of motion for the mechanical system errors on the sliding surface are given by $\dot{\mathbf{e}}=\varepsilon$,

$\dot{\varepsilon}=-A \operatorname{sig}(\mathbf{e})^{\rho_{1}}-B \operatorname{sig}(\varepsilon)^{\rho_{2}}$,

and the definition of $\mathbf{u}_{0}$ is

$\mathbf{u}_{0}=-\mathbf{f}_{e}+\dot{\varepsilon}=-\mathbf{f}_{e}-A \operatorname{sig}(\mathbf{e})^{\rho_{1}}-B \operatorname{sig}(\varepsilon)^{\rho_{2}}$.

From Theorem 2, the finite-time convergence of (13) is guaranteed. Thus, if the system is on the sliding surface (disturbance $\mathbf{d}=\mathbf{0}$ ), then the errors $\mathbf{e}$ and $\varepsilon$ in (13) will converge to zero in finite time and the control vector $\mathbf{u}_{0}$ required is given by (14).

Next, we define a control vector $\mathbf{u}_{s}$ so that the combined control $\mathbf{u}=\mathbf{u}_{0}+\mathbf{u}_{s}$ is a smooth SOSMC that will drive the mechanical system (10) subject to a disturbance $\mathbf{d} \neq \mathbf{0}_{n}$ onto the sliding surface $\mathbf{s}=\mathbf{0}_{n}$ in finite time and then drive the errors on the sliding surface to a neighborhood of $\mathbf{0}_{n}$ in finite time.

We will prove in Theorem 3 that the following choice for $\mathbf{u}_{s}$ gives the required finite-time convergence.

$\begin{aligned} \mathbf{u}_{s} & =-H \operatorname{sig}(\mathbf{s})^{\frac{(\alpha+1)}{2}}-K \mathbf{s}+\gamma, \\ \dot{\gamma} & =-N \operatorname{sig}(\mathbf{s})^{\alpha}-P \mathbf{s}\end{aligned}$

where $\alpha \in(0,1)$ and

$H=\operatorname{diag}\left(h_{i}>0\right) \in \Re^{n \times n}, \quad K=\operatorname{diag}\left(k_{i}>0\right) \in \mathfrak{R}^{n \times n}$,

$N=\operatorname{diag}\left(n_{i}>0\right) \in \mathfrak{R}^{n \times n}, \quad P=\operatorname{diag}\left(p_{i}>0\right) \in \mathfrak{R}^{n \times n}$.

Therefore, combining (14) and (15), we obtain the proposed smooth SOSMC control law as

$\mathbf{u}=-\mathbf{f}_{e}-A \operatorname{sig}(\mathbf{e})^{\rho_{1}}-B \operatorname{sig}(\varepsilon)^{\rho_{2}}-H \operatorname{sig}(\mathbf{s})^{\frac{(\alpha+1)}{2}}-K \mathbf{s}+\gamma$,

$\dot{\gamma}=-N \operatorname{sig}(\mathbf{s})^{\alpha}-P \mathbf{s}$.

Note that the initial conditions $\mathbf{s}(0)$ and $\dot{\mathbf{s}}(0)$ can be nonzero since we are assuming that the disturbances $\mathbf{d}$ and $\mathbf{d}$ can initially be non-zero, i.e., the system is not initially on the sliding surface.

For the disturbed system (10) and control (17), the time dependence of $\mathbf{s}$ is given by

$$
\begin{aligned}
\dot{\mathbf{s}}(t) & =\dot{\boldsymbol{\varepsilon}}+A \operatorname{sig}(\mathbf{e}(t))^{\rho_{1}}+B \operatorname{sig}(\varepsilon(t))^{\rho_{2}}, \\
& =\mathbf{u}+\mathbf{f}_{e}+\mathbf{d}+A \operatorname{sig}(\mathbf{e}(t))^{\rho_{1}}+B \operatorname{sig}(\varepsilon(t))^{\rho_{2},} \\
& =-H \operatorname{sig}(\mathbf{s})^{\frac{(\alpha+1)}{2}}-K \mathbf{s}+\gamma+\mathbf{d},
\end{aligned}
$$

where

$\dot{\gamma}=-N \operatorname{sig}(\mathbf{s})^{\alpha}-P \mathbf{s}$.

We now prove that the control law $\mathbf{u}=\mathbf{u}_{0}+\mathbf{u}_{s}$ in (17) will drive the disturbed system (18) into a neigborhood of the sliding surface in finite time. 
Theorem 3 If the control law is defined as (17) and the positive gains $h_{i}, k_{i}, n_{i}$ and $p_{i},(i=1,2, \ldots, n)$ in (16) are chosen such that the conditions

$n_{i}>h_{i}^{2} \frac{(\alpha+1)^{2}}{4}, \quad n_{i} p_{i}>h_{i}^{2} p_{i} \frac{(\alpha+1)^{2}}{4}+h_{i}^{2} k_{i}^{2} \frac{(\alpha+3)^{2}}{4}$

are satisfied, then the trajectory $\mathbf{s}(t)$ in (18) converges to the neighborhood of zero in finite time.

Proof In (18), let $\mathbf{z}=\left[\begin{array}{llll}z_{1} & z_{2} & \ldots & z_{n}\end{array}\right]^{T}=\mathbf{s} \in \mathfrak{R}^{n}$ and $\xi=\left[\begin{array}{llll}\xi_{1} & \xi_{2} & \ldots & \xi_{n}\end{array}\right]^{T}=\boldsymbol{\gamma}+\mathbf{d} \in \mathfrak{R}^{n}$. Then, we have

$\dot{\mathbf{z}}=-H \operatorname{sig}(\mathbf{z})^{\frac{(\alpha+1)}{2}}-K \mathbf{z}+\xi$,

$\dot{\xi}=-N \operatorname{sig}(\mathbf{z})^{\alpha}-P \mathbf{z}+\phi$,

where $\phi=\dot{d}$.

Since the matrices $H, K, N$ and $P$ in (17) are assumed to be diagonal, the system of Eq. (20) can be considered independently for each component $z_{i}$ and $\xi_{i}$. Then, from (20), and using (11) and (16), we have

$\dot{z}_{i}=-h_{i} \operatorname{sig}\left(z_{i}\right)^{\frac{(\alpha+1)}{2}}-K z_{i}+\xi_{i}=-h_{i}\left|z_{i}\right|^{\frac{(\alpha+1)}{2}} \operatorname{sgn}\left(z_{i}\right)-K z_{i}+\xi_{i}$, $\dot{\xi}_{i}=-n_{i} \operatorname{sig}\left(z_{i}\right)^{\alpha}-p_{i} z_{i}+\phi_{i}=-n_{i}\left|z_{i}\right|^{\alpha} \operatorname{sgn}\left(z_{i}\right)-p_{i} z_{i}+\phi_{i}$,

where $\phi_{i}=\dot{d}_{i}$.

We will first use a Lyapunov function to prove global convergence of the components $z_{i}, \xi_{i}$ to the neighborhood of the origin, and then prove finite-time convergence by proving that the conditions of Lemma 1 are satisfied for the Lyapunov function.

Let a Lyapunov candidate function be defined as

$V=\sum_{i=1}^{n} V_{i}$

where

$V_{i}=\frac{2 n_{i}}{\alpha+1}\left|z_{i}\right|^{\alpha+1}+p_{i}\left|z_{i}\right|^{2}+\frac{1}{2}\left|\xi_{i}\right|^{2}+\frac{1}{2}\left|\psi_{i}\right|^{2}$,

and

$\psi_{i}=-\xi_{i}+h_{i}\left|z_{i}\right|^{\frac{\alpha-1}{2}} z_{i}+k_{i} z_{i}$.

It is clear that the functions $V_{i}$ are positive definite and have unique minima at $\left(z_{i}, \xi_{i}\right)=(0,0)$. However, to prove finite-time convergence using Lemma 1 it is necessary to transform the $V_{i}$ into positive-definite matrix forms.

Equations (23) and (24) can be written in the following matrix form as

$V_{i}=\Gamma_{i}^{T} \Pi_{i} \Gamma_{i}$

$$
\Gamma_{i}=\left[\begin{array}{c}
\left|z_{i}\right|^{\frac{\alpha-1}{2}} z_{i} \\
z_{i} \\
\xi_{i}
\end{array}\right] \text { and } \Pi_{i}=\frac{1}{2}\left[\begin{array}{ccc}
\left(\frac{4 n_{i}}{\alpha+1}+h_{i}^{2}\right) & h_{i} k_{i} & -h_{i} \\
h_{i} k_{i} & \left(2 p_{i}+k_{i}^{2}\right) & -k_{i} \\
-h_{i} & -k_{i} & 2
\end{array}\right] \text {. }
$$

Further, the matrix $\Pi_{i}$ is positive definite since its principal minors are positive as shown below:

$\Delta_{11}=\frac{2 n_{i}}{\alpha+1}+\frac{1}{2} h_{i}^{2}>0, \Delta_{12}=\frac{2 n_{i}}{\alpha+1}\left(2 p_{i}+k_{i}^{2}\right)+h_{i}^{2} p_{i}>0$,

$\Delta_{13}=\frac{4 n_{i}}{\alpha+1}+\frac{1}{2} h_{i}^{2}>0, \Delta_{23}=\frac{1}{2} k_{i}^{2}+2 p_{i}>0$,

$\Delta_{33}=\frac{2 n_{i}}{\alpha+1}\left(4 p_{i}+k_{i}^{2}\right)+h_{i}^{2} p_{i}>0$.

Thus, the $V_{i}$ can be written in positive-definite matrix forms with unique minima $V_{i}(0)=0$. Also, since $\Pi_{i}$ is positive definite, all eigenvalues of $\Pi_{i}$ are real and positive.

We now prove that the system (20) is globally asymptotically convergent by proving that the time derivatives of the $V_{i}$ are negative definite.

Taking the derivative of (23), we obtain

$$
\begin{aligned}
\dot{V}_{i}= & \left(\frac{2 n_{i}}{\alpha+1}+\frac{1}{2} h_{i}^{2}\right) \frac{d}{d t}\left(\left|z_{i}\right|^{\alpha-1} z_{i}^{2}\right)+\left(p_{i}+\frac{1}{2} k_{i}^{2}\right) \frac{d}{d t}\left(z_{i}^{2}\right)+\frac{d}{d t}\left(\xi_{i}^{2}\right) \\
& +h_{i} k_{i} \frac{d}{d t}\left(\left|z_{i}\right|^{\frac{\alpha-1}{2}} z_{i}^{2}\right)-h_{i} \frac{d}{d t}\left(\left|z_{i}\right|^{\frac{\alpha-1}{2}} z_{i} \xi_{i}\right)-k_{i} \frac{d}{d t}\left(z_{i} \xi_{i}\right) \\
= & \left(\frac{4 n_{i}}{\alpha+1}+h_{i}^{2}\right)\left|z_{i}\right|^{\alpha-1} z_{i} \dot{z}_{i}+\left(\frac{2 n_{i}}{\alpha+1}+\frac{1}{2} h_{i}^{2}\right)(\alpha-1)\left|z_{i}\right|^{\alpha-3} z_{i}^{3} \dot{z}_{i} \\
& +\left(2 p_{i}+k_{i}^{2}\right) z_{i} \dot{z}_{i}+2 \xi_{i} \dot{\xi}_{i}+2 h_{i} k_{i}\left|z_{i}\right|^{\frac{\alpha-1}{2}} z_{i} \dot{z}_{i}+\frac{(\alpha-1)}{2} h_{i} k_{i}\left|z_{i}\right|^{\frac{\alpha-5}{2}} z_{i}^{3} \dot{z}_{i} \\
& -h_{i} \frac{(\alpha-1)}{2}\left|z_{i}\right|^{\frac{\alpha-5}{2}} z_{i}^{2} \xi_{i} \dot{z}_{i}-h_{i}\left|z_{i}\right|^{\frac{\alpha-1}{2}} \dot{z}_{i} \xi_{i}-h_{i}\left|z_{i}\right|^{\frac{\alpha-1}{2}} z_{i} \dot{\xi}_{i} \\
& -k_{i} z_{i} \dot{\xi}_{i}-k_{i} \dot{z}_{i} \xi_{i} .
\end{aligned}
$$

Then, substituting (20) into (28), we have

$$
\begin{aligned}
\dot{V}_{i}= & \left(\frac{4 n_{i}}{\alpha+1}+h_{i}^{2}\right)\left|z_{i}\right|^{\alpha-1} z_{i}\left(\xi_{i}-h_{i}\left|z_{i}\right|^{\frac{\alpha+1}{2}} \operatorname{sgn}\left(z_{i}\right)-k_{i} z_{i}\right) \\
& +\left(\frac{2 n_{i}}{\alpha+1}+\frac{1}{2} h_{i}^{2}\right)(\alpha-1)\left|z_{i}\right|^{\alpha-3} z_{i}^{3}\left(\xi_{i}-h_{i}\left|z_{i}\right|^{\frac{\alpha+1}{2}} \operatorname{sgn}\left(z_{i}\right)-k_{i} z_{i}\right) \\
& +\left(2 p_{i}+k_{i}^{2}\right) z_{i}\left(\xi_{i}-h_{i}\left|z_{i}\right|^{\frac{\alpha+1}{2}} \operatorname{sgn}\left(z_{i}\right)-k_{i} z_{i}\right) \\
& +2 \xi_{i}\left(-n_{i}\left|z_{i}\right|^{\alpha} \operatorname{sgn}\left(z_{i}\right)-p_{i} z_{i}+\phi_{i}\right) \\
& +2 h_{i} k_{i}\left|z_{i}\right|^{\frac{\alpha-1}{2}} z_{i}\left(\xi_{i}-h_{i}\left|z_{i}\right|^{\frac{\alpha+1}{2}} \operatorname{sgn}\left(z_{i}\right)-k_{i} z_{i}\right) \\
& +\frac{(\alpha-1)}{2} h_{i} k_{i}\left|z_{i}\right|^{\frac{\alpha-5}{2}} z_{i}^{3}\left(\xi_{i}-h_{i}\left|z_{i}\right|^{\frac{\alpha+1}{2}} \operatorname{sgn}\left(z_{i}\right)-k_{i} z_{i}\right) \\
& -h_{i} \frac{(\alpha-1)}{2}\left|z_{i}\right|^{\frac{\alpha-5}{2}} z_{i}^{2} \xi_{i}\left(\xi_{i}-h_{i}\left|z_{i}\right|^{\frac{\alpha+1}{2}} \operatorname{sgn}\left(z_{i}\right)-k_{i} z_{i}\right) \\
& -h_{i}\left|z_{i}\right|^{\frac{\alpha-1}{2}}\left(\xi_{i}-h_{i}\left|z_{i}\right|^{\frac{\alpha+1}{2}} \operatorname{sgn}\left(z_{i}\right)-k_{i} z_{i}\right) \xi_{i} \\
& -h_{i}\left|z_{i}\right|^{\frac{\alpha-1}{2}} z_{i}\left(-n_{i}\left|z_{i}\right|^{\alpha} \operatorname{sgn}\left(z_{i}\right)-p_{i} z_{i}+\phi_{i}\right) \\
& -k_{i} z_{i}\left(-n_{i}\left|z_{i}\right|^{\alpha} \operatorname{sgn}\left(z_{i}\right)-p_{i} z_{i}+\phi_{i}\right) \\
& -k_{i}\left(\xi_{i}-h_{i}\left|z_{i}\right|^{\frac{\alpha+1}{2}} \operatorname{sgn}\left(z_{i}\right)-k_{i} z_{i}\right) \xi_{i} .
\end{aligned}
$$

where 
We can expand Eq. (29) and obtain

$$
\begin{aligned}
\dot{V}_{i}= & \left(\frac{4 n_{i}}{\alpha+1}+h_{i}^{2}\right)\left|z_{i}\right|^{\alpha-1} z_{i} \xi_{i}-h_{i}\left(\frac{4 n_{i}}{\alpha+1}+h_{i}^{2}\right)\left|z_{i}\right|^{\alpha-1}\left|z_{i}\right|^{\frac{\alpha+1}{2}} \operatorname{sgn}\left(z_{i}\right) z_{i} \\
& -k_{i}\left(\frac{4 n_{i}}{\alpha+1}+h_{i}^{2}\right)\left|z_{i}\right|^{\alpha-1} z_{i} z_{i}+(\alpha-1)\left(\frac{2 n_{i}}{\alpha+1}+\frac{1}{2} h_{i}^{2}\right)\left|z_{i}\right|^{\alpha-3} z_{i}^{3} \xi_{i} \\
& -(\alpha-1) h_{i}\left(\frac{2 n_{i}}{\alpha+1}+\frac{1}{2} h_{i}^{2}\right)\left|z_{i}\right|^{\alpha-3}\left|z_{i}\right|^{\frac{\alpha+1}{2}} \operatorname{sgn}\left(z_{i}\right) z_{i}^{3} \\
& -(\alpha-1) k_{i}\left(\frac{2 n_{i}}{\alpha+1}+\frac{1}{2} h_{i}^{2}\right)\left|z_{i}\right|^{\alpha-3} z_{i}^{4}+\left(2 p_{i}+k_{i}^{2}\right) z_{i} \xi_{i} \\
& -\left(2 h_{i} p_{i}+h_{i} k_{i}^{2}\right)\left|z_{i}\right|^{\frac{\alpha+1}{2}} \operatorname{sgn}\left(z_{i}\right) z_{i}-\left(2 k_{i} p_{i}+k_{i}^{3}\right) z_{i}^{2}-2 n_{i}\left|z_{i}\right|^{\alpha} \operatorname{sgn}\left(z_{i}\right) \xi_{i} \\
& -2 p_{i} \xi_{i} z_{i}+2 \xi_{i} \phi_{i}+2 h_{i} k_{i}\left|z_{i}\right|^{\frac{\alpha-1}{2}} z_{i} \xi_{i}-2 h_{i}^{2} k_{i}\left|z_{i}\right|^{\frac{\alpha-1}{2}}\left|z_{i}\right|^{\frac{\alpha+1}{2}} \operatorname{sgn}\left(z_{i}\right) z_{i} \\
& -2 h_{i} k_{i}^{2}\left|z_{i}\right|^{\frac{\alpha-1}{2}} z_{i} z_{i}+\frac{(\alpha-1)}{2} h_{i} k_{i}\left|z_{i}\right|^{\frac{\alpha-5}{2}} z_{i}^{3} \xi_{i}-\frac{(\alpha-1)}{2} h_{i}^{2} k_{i}\left|z_{i}\right|^{\frac{\alpha-5}{2}}\left|z_{i}\right|^{\frac{\alpha+1}{2}} \operatorname{sgn}\left(z_{i}\right) z_{i}^{3} \\
& -\frac{(\alpha-1)}{2} h_{i} k_{i}^{2}\left|z_{i}\right|^{\frac{\alpha-5}{2}} z_{i}^{4}-\frac{(\alpha-1)}{2} h_{i}\left|z_{i}\right|^{\frac{\alpha-5}{2}} z_{i}^{2} \xi_{i}^{2}+\frac{(\alpha-1)}{2} h_{i}^{2}\left|z_{i}\right|^{\frac{\alpha-5}{2}}\left|z_{i}\right|^{\frac{\alpha+1}{2}} \operatorname{sgn}\left(z_{i}\right) z_{i}^{2} \xi_{i} \\
& +\frac{(\alpha-1)}{2} h_{i} k_{i}\left|z_{i}\right|^{\frac{\alpha-5}{2}} z_{i}^{2} \xi_{i} z_{i}-h_{i}\left|z_{i}\right|^{\frac{\alpha-1}{2}} \xi_{i}^{2}+h_{i}^{2}\left|z_{i}\right|^{\frac{\alpha-1}{2}}\left|z_{i}\right|^{\frac{\alpha+1}{2}} \operatorname{sgn}\left(z_{i}\right) \xi_{i} \\
& +h_{i} k_{i}\left|z_{i}\right|^{\frac{\alpha-1}{2}} z_{i} \xi_{i}+h_{i} n_{i}\left|z_{i}\right|^{\frac{\alpha-1}{2}}\left|z_{i}\right|^{\alpha} \operatorname{sgn}\left(z_{i}\right) z_{i}+h_{i} p_{i}\left|z_{i}\right|^{\frac{\alpha-1}{2}} z_{i}^{2}-h_{i}\left|z_{i}\right|^{\frac{\alpha-1}{2}} z_{i} \phi_{i} \\
& +k_{i} n_{i}\left|z_{i}\right|^{\alpha} \operatorname{sgn}\left(z_{i}\right) z_{i}+k_{i} p_{i} z_{i} z_{i}-k_{i} z_{i} \phi_{i} \\
& -k_{i} \xi_{i} \xi_{i}+k_{i} h_{i}\left|z_{i}\right|^{\frac{\alpha+1}{2}} \operatorname{sgn}\left(z_{i}\right) \xi_{i}+k_{i} k_{i} z_{i} \xi_{i} .
\end{aligned}
$$

Next, using $\operatorname{sgn}\left(z_{i}\right)=\frac{z_{i}}{\left|z_{i}\right|}$ and simplifying the equation, we obtain

$$
\begin{aligned}
\dot{V}_{i}= & \left(\frac{2 n_{i}}{\alpha+1}+h_{i}^{2}\right)\left|z_{i}\right|^{\alpha-1} z_{i} \xi_{i}+\left(\frac{2 n_{i}}{\alpha+1}+\frac{1}{2} h_{i}^{2}\right)(\alpha-1)\left|z_{i}\right|^{\alpha-1} z_{i} \xi_{i} \\
& -2 n_{i}\left|z_{i}\right|^{\alpha-1} z_{i} \xi_{i}+h_{i}^{2}\left|z_{i}\right|^{\alpha-1} z_{i} \xi_{i}+\frac{(\alpha-1)}{2} h_{i}^{2}\left|z_{i}\right|^{\alpha-1} z_{i} \xi_{i} \\
& -\left(\frac{2 k_{i} n_{i}}{\alpha+1}+\frac{1}{2} h_{i}^{2} k_{i}\right)(\alpha-1)\left|z_{i}\right|^{\alpha-1} z_{i} z_{i}-\left(\frac{4 k_{i} n_{i}}{\alpha+1}+h_{i}^{2} k_{i}\right)\left|z_{i}\right|^{\alpha-1} z_{i} z_{i}+k_{i} n_{i}\left|z_{i}\right|^{\alpha-1} z_{i}^{2} \\
& -\left(\frac{4 h_{i} n_{i}}{\alpha+1}+h_{i}^{3}\right)\left|z_{i}\right|^{\frac{\alpha-1}{2}}\left|z_{i}\right|^{\alpha-1} z_{i} z_{i}-\left(\frac{2 h_{i} n_{i}}{\alpha+1}+\frac{1}{2} h_{i}^{3}\right)(\alpha-1)\left|z_{i}\right|^{\frac{\alpha-1}{2}}\left|z_{i}\right|^{\alpha-1} z_{i}^{2} \\
& +h_{i} n_{i}\left|z_{i}\right|^{\frac{\alpha-1}{2}}\left|z_{i}\right|^{\alpha-1} z_{i}^{2}+\left(2 p_{i}+k_{i}^{2}\right) z_{i} \xi_{i}+k_{i}^{2} z_{i} \xi_{i}-2 p_{i} \xi_{i} z_{i} \\
& +2 h_{i} k_{i}\left|z_{i}\right|^{\frac{\alpha-1}{2}} z_{i} \xi_{i}+h_{i} k_{i}\left|z_{i}\right|^{\frac{\alpha-1}{2}} z_{i} \xi_{i}+\frac{(\alpha-1)}{2} h_{i} k_{i}\left|z_{i}\right|^{\frac{\alpha-1}{2}} z_{i} \xi_{i}+h_{i} k_{i}\left|z_{i}\right|^{\frac{\alpha-1}{2}} z_{i} \xi_{i} \\
& +\frac{(\alpha-1)}{2} h_{i} k_{i}\left|z_{i}\right|^{\frac{\alpha-1}{2}} \xi_{i} z_{i}-\left(2 k_{i} p_{i}+k_{i}^{3}\right) z_{i}^{2}+k_{i} p_{i} z_{i} z_{i}-2 h_{i}^{2} k_{i}\left|z_{i}\right|^{\alpha-1} z_{i}^{2} \\
& -\frac{(\alpha-1)}{2} h_{i}^{2} k_{i}\left|z_{i}\right|^{\alpha-1} z_{i}^{2}-\left(2 h_{i} p_{i}+h_{i} k_{i}^{2}\right)\left|z_{i}\right|^{\frac{\alpha-1}{2}} z_{i}^{2}-2 h_{i} k_{i}^{2}\left|z_{i}\right|^{\frac{\alpha-1}{2}} z_{i} z_{i} \\
& -\frac{(\alpha-1)}{2} h_{i} k_{i}^{2}\left|z_{i}\right|^{\frac{\alpha-1}{2}} z_{i}^{2}+h_{i} p_{i}\left|z_{i}\right|^{\frac{\alpha-1}{2}} z_{i}^{2}-\frac{(\alpha-1)}{2} h_{i}\left|z_{i}\right|^{\frac{\alpha-1}{2}} \xi_{i}^{2} \\
& -h_{i}\left|z_{i}\right|^{\frac{\alpha-1}{2}} \xi_{i}^{2}-k_{i} \xi_{i} \xi_{i}-h_{i}\left|z_{i}\right|^{\frac{\alpha-1}{2}} z_{i} \phi_{i}-k_{i} z_{i} \phi_{i}+2 \xi_{i} \phi_{i} .
\end{aligned}
$$


Finally, we have

$$
\begin{aligned}
\dot{V}_{i}= & -\left|z_{i}\right|^{\frac{\alpha-1}{2}}\left\{\left(h_{i} n_{i}+\frac{(\alpha+1)}{2} h_{i}^{3}\right)\left|z_{i}\right|^{\alpha-1} z_{i}^{2}+\left(h_{i} p_{i}+\frac{(\alpha+5)}{2} h_{i} k_{i}^{2}\right) z_{i}^{2}\right. \\
& \left.-(\alpha+1) h_{i}^{2}\left|z_{i}\right|^{\frac{\alpha-1}{2}} z_{i} \xi_{i}+\frac{(\alpha+1)}{2} h_{i} \xi_{i}^{2}\right\} \\
& -\left\{\left(k_{i} n_{i}+(\alpha+2) h_{i}^{2} k_{i}\right)\left|z_{i}\right|^{\alpha-1} z_{i} z_{i}+\left(k_{i} p_{i}+k_{i}^{3}\right) z_{i}^{2}\right. \\
& \left.-(\alpha+3) h_{i} k_{i}\left|z_{i}\right|^{\frac{\alpha-1}{2}} z_{i} \xi_{i}-2 k_{i}^{2} z_{i} \xi_{i}+k_{i} \xi_{i} \xi_{i}\right\} \\
& -\left(h_{i}\left|z_{i}\right|^{\frac{\alpha-1}{2}} z_{i}+k_{i} z_{i}-2 \xi_{i}\right) \phi_{i} .
\end{aligned}
$$

Equation (32) can then be rewritten in the matrix form:

$\dot{V}_{i}=-\left|z_{i}\right|^{\frac{\alpha-1}{2}} \Gamma_{i}^{T} Q_{i} \Gamma_{i}-\Gamma_{i}^{T} R_{i} \Gamma_{i}-\Gamma_{i}^{T} \varsigma_{i} \phi_{i}$,

where $\Gamma_{i}$ is defined in (26), $\varsigma_{i}=\left[h_{i} k_{i}-2\right]^{T}$, and the matrices $Q_{i}$ and $R_{i}$ are given by

$Q_{i}=\left[\begin{array}{ccc}h_{i}\left(n_{i}+h_{i}^{2} \frac{(\alpha+1)}{2}\right) & 0 & -h_{i}^{2} \frac{(\alpha+1)}{2} \\ 0 & h_{i}\left(p_{i}+k_{i}^{2} \frac{(\alpha+5)}{2}\right) & 0 \\ -h_{i}^{2} \frac{(\alpha+1)}{2} & 0 & h_{i} \frac{(\alpha+1)}{2}\end{array}\right]$,

and

$R_{i}=\left[\begin{array}{ccc}k_{i}\left(n_{i}+h_{i}^{2}(\alpha+2)\right) & 0 & -h_{i} k_{i} \frac{(\alpha+3)}{2} \\ 0 & k_{i}\left(p_{i}+k_{i}^{2}\right) & -k_{i}^{2} \\ -h_{i} k_{i} \frac{(\alpha+3)}{2} & -k_{i}^{2} & k_{i}\end{array}\right]$.

Next, we find conditions for the $Q_{i}$ and $R_{i}$ to be positivedefinite matrices by checking their principal minors. The principal minors of $Q_{i}$ are

$$
\begin{aligned}
& \Delta_{11}=h_{i}\left(n_{i}+h_{i}^{2} \frac{(\alpha+1)}{2}\right)>0, \\
& \Delta_{12}=h_{i}^{2}\left(n_{i}+h_{i}^{2} \frac{(\alpha+1)}{2}\right)\left(p_{i}+k_{i}^{2} \frac{(\alpha+5)}{2}\right)>0, \\
& \Delta_{13}=h_{i}^{2} n_{i} \frac{(\alpha+1)}{2}>0, \\
& \Delta_{23}=h_{i}^{2} \frac{(\alpha+1)}{2}\left(p_{i}+k_{i}^{2} \frac{(\alpha+5)}{2}\right)>0, \\
& \Delta_{33}=h_{i}^{3} n_{i} p_{i} \frac{(\alpha+1)}{2}+h_{i}^{3} k_{i}^{2} n_{i} \frac{(\alpha+1)}{4}(\alpha+5)>0 .
\end{aligned}
$$

Thus, $Q_{i}$ is positive definite for all positive values of $h_{i}, k_{i}, n_{i}$ and $p_{i}$. The principal minors of $R_{i}$ are
$\Delta_{11}=k_{i}\left(n_{i}+h_{i}^{2}(\alpha+2)\right)>0$,

$\Delta_{12}=k_{i}^{2}\left(n_{i}+h_{i}^{2}(\alpha+2)\right)\left(p_{i}+k_{i}^{2}\right)>0$,

$\Delta_{13}=k_{i}^{2}\left(n_{i}-h_{i}^{2} \frac{(\alpha+1)^{2}}{4}\right)$,

$\Delta_{23}=k_{i}^{2} p_{i}>0$,

$\Delta_{33}=k_{i}^{3}\left(n_{i} p_{i}-h_{i}^{2} p_{i} \frac{(\alpha+1)^{2}}{4}-h_{i}^{2} k_{i}^{2} \frac{(\alpha+3)^{2}}{4}\right)$.

Therefore, by choosing the parameters $h_{i}, k_{i}, n_{i}$ and $p_{i}$ to satisfy the conditions in (19), the principal minors $\Delta_{13}>0$ and $\Delta_{33}>0$, and hence $R_{i}$ is also positive definite.

From Eq. (26), we have

$\left\|\Gamma_{i}\right\|^{2}=\Gamma_{i}^{T} \Gamma_{i}=\left|z_{i}\right|^{\alpha-1} z_{i}^{T} z_{i}+z_{i}^{T} z_{i}+\xi_{i}^{T} \xi_{i} \geq\left|z_{i}\right|^{2}$,

and therefore, since $\alpha-1<0$, we obtain $\|\left.\Gamma_{i}\right|^{\frac{\alpha-1}{2}} \leq\left|z_{i}\right|^{\frac{\alpha-1}{2}}$.

Thus, we can rewrite (33) as

$\dot{V}_{i} \leq-\left\|\Gamma_{i}\right\|^{\frac{\alpha-1}{2}} \lambda_{\min }\left(Q_{i}\right)\left\|\Gamma_{i}\right\|^{2}-\lambda_{\min }\left(R_{i}\right)\left\|\Gamma_{i}\right\|^{2}+\left|\phi_{i}\right|\left\|s_{i}\right\|\left\|\Gamma_{i}\right\|$.

Using the assumption $\left|\phi_{i}\right| \leq \bar{\phi}_{i}$, we obtain

$\dot{V}_{i} \leq-\lambda_{\min }\left(Q_{i}\right)\left\|\Gamma_{i}\right\|^{\frac{\alpha+3}{2}}-\lambda_{\min }\left(R_{i}\right)\left\|\Gamma_{i}\right\|^{2}+\bar{\phi}_{i}\left\|\varsigma_{i}\right\| \Gamma_{i} \|$.

Then, from Eq. (25), we have $\frac{V_{i}}{\lambda_{\max }\left(\Pi_{i}\right)} \leq\left\|\Gamma_{i}\right\|^{2}$, and therefore

$$
\begin{aligned}
\dot{V}_{i} & \leq-\lambda_{\min }\left(Q_{i}\right)\left(\frac{V_{i}}{\lambda_{\max }\left(\Pi_{i}\right)}\right)^{\frac{\alpha+3}{4}}-\lambda_{\min }\left(R_{i}\right) \frac{V_{i}}{\lambda_{\max }\left(\Pi_{i}\right)}+\bar{\phi}_{i}\left\|\varsigma_{i}\right\|\left\|\Gamma_{i}\right\| \\
& =-\frac{\lambda_{\min }\left(Q_{i}\right)}{\lambda_{\max }\left(\Pi_{i}\right)^{\frac{\alpha+3}{4}}} V_{i}^{\frac{\alpha+3}{4}}-\frac{\lambda_{\min }\left(R_{i}\right)}{\lambda_{\max }\left(\Pi_{i}\right)} V_{i}+\bar{\phi}_{i}\left\|\varsigma_{i}\right\|\left\|\Gamma_{i}\right\|, \\
& =-\beta_{i} V_{i}^{\eta}-\delta_{i} V_{i}+\bar{\phi}_{i}\left\|\varsigma_{i}\right\|\left\|\Gamma_{i}\right\|,
\end{aligned}
$$

where

$\beta_{i}=\frac{\lambda_{\text {min }}\left(Q_{i}\right)}{\lambda_{\max }\left(\Pi_{i}\right)^{\frac{\alpha+3}{4}}}, \quad \delta_{i}=\frac{\lambda_{\min }\left(R_{i}\right)}{\lambda_{\max }\left(\Pi_{i}\right)^{\prime}}, \quad \eta=\frac{\alpha+3}{4}<1$.

For $\theta \in(0,1)$, we can rewrite (39) as

$\dot{V}_{i} \leq-\theta \beta_{i} V_{i}^{\eta}-(1-\theta) \beta_{i} V_{i}^{\eta}-\delta_{i} V_{i}+\bar{\phi}_{i}\left\|\varsigma_{i}\right\|\left\|\Gamma_{i}\right\|$.

Thus,

$\dot{V}_{i} \leq-\theta \beta_{i} V_{i}^{\eta}-\delta_{i} V_{i}$

whenever

$\left\|\Gamma_{i}\right\| \geq\left(\frac{\bar{\phi}_{i}\left\|\varsigma_{i}\right\|}{\beta_{i}(1-\theta) \lambda_{\min }\left(\Pi_{i}\right)^{\eta}}\right)^{\frac{2}{(\alpha+1)}}$ 
The differential inequality in (42) is a special case of the differential inequality (2) in Lemma 1 . Hence, if the matrices $H, K, N$ and $P$ are selected satisfying the conditions in (19), each component of the system (20) will converge into a region

$\left\|\Gamma_{i}\right\| \leq \bar{\Gamma}_{i}=\left(\frac{\bar{\phi}_{i}\left\|s_{i}\right\|}{\beta_{i}(1-\theta) \lambda_{\min }\left(\Pi_{i}\right)^{\eta}}\right)^{\frac{2}{(\alpha+1)}}$

in finite time.

From Eq. (3), each component should converge in the finite time

$T_{i}=t_{0}+\frac{1}{\delta_{i}(1-\eta)} \ln \left(\frac{\delta_{i} V_{i}\left(t_{0}\right)^{1-\eta}+\theta \beta_{i}}{\theta \beta_{i}}\right)$,

and therefore all components will converge in the finite time $T_{f}=\max \left(T_{i}\right)$.

Therefore, we have proved that the trajectory $s(t)$ in (12) with the control law (17) will converge to the neighborhood of the sliding surface $s$ in (12) in finite time.

In this section, we have proved that the smooth SOSMC that we have proposed will drive a disturbed system given by Eqs. (9) and (10) into a neigborhood of the sliding surface $\boldsymbol{s}$ in (12) in finite time and then drive the system along the sliding surface to a desired trajectory $\left(\mathbf{q}_{d}, \mathbf{v}_{d}\right)$ in finite time.

\section{Simulation study}

In this section, we illustrate the effectiveness of the proposed smooth SOSMC for finite-time control of mechanical systems. As a first simple example, we illusrate the effectiveness of the smooth SOSMC on the one-dimensional system of the variable-length pendulum [40] and compare its effectiveness with a super-twisting (STW) algorithm [16]. We then test the finite-time convergence and

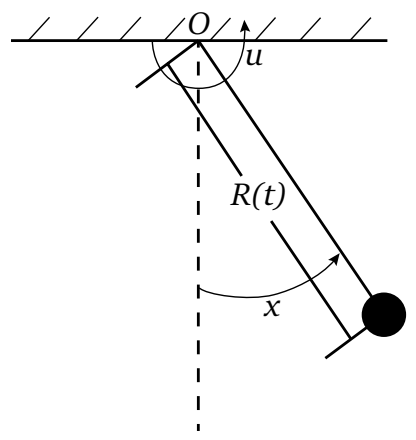

Fig. 1 The variable-length pendulum [48]

\section{SN Applied Sciences}

chattering properties of the smooth SOSMC on a multivariable system, namely the two-link robot manipulator [41], and compare its behavior with an STW algorithm [16] and a super-twisting like (STL) algorithm [17]. Since both the STW and STL controls are discontinuous, we have used Euler's method with step size 0.0001 to integrate the equations of motion for the smooth SOSMC, STW and STL methods. The step size of 0.0001 was selected to obtain reasonable accuracy in the numerical studies.

\subsection{Numerical results for variable-length pendulum}

We have carried out simulations on the variable-length pendulum shown in Fig. 1.

The equation of motion for the variable-length pendulum with disturbance $R$ can be written as $[40,48]$

$\ddot{x}=-2 \frac{\dot{R}}{R} \dot{x}-g \frac{1}{R} \sin (x)+\frac{1}{m R^{2}} u ，$

where $x$ is the oscillation angle $(\mathrm{rad}), g=9.81 \mathrm{~m} \cdot \mathrm{s}^{-2}$ is the acceleration due to gravity, $m$ is the mass of the pendulum $(\mathrm{kg}), R$ is the distance $(\mathrm{m})$ from the axis of rotation of the pendulum to the mass, and $u$ is the control input $(N \cdot m)$.

Equation (46) can be rewritten as the first-order differential system:

$\dot{q}=v$,

$\dot{v}=-2 \frac{\dot{R}}{R} v-g \frac{1}{R} \sin (q)+\frac{1}{m R^{2}} u$,

where $q=x(\mathrm{rad})$ and $v=\dot{x}\left(\mathrm{rad} \cdot \mathrm{s}^{-1}\right)$.

We then define the smooth second-order sliding surface as

$s=\varepsilon+\int\left(a|e|^{\rho_{1}} \operatorname{sgn}(e)+b|\varepsilon|^{\rho_{2}} \operatorname{sgn}(\varepsilon)\right) d t=0$,

$\left.\dot{s}=\dot{\varepsilon}+a|e|^{\rho_{1}} \operatorname{sgn}(e)+b|\varepsilon|^{\rho_{2}} \operatorname{sgn}(\varepsilon)\right)=0$,

and then the control $u$ in Eq. (46) is given by

$u=m R^{2}\left[2 \frac{\dot{R}}{R} v+g \frac{1}{R} \sin (q)-a|e|^{\rho_{1}} \operatorname{sgn}(e)-b|\varepsilon|^{\rho_{2}} \operatorname{sgn}(\varepsilon)+u_{s}\right]$.

The equivalent control for the smooth SOSMC is then

$u_{s}=-h|s|^{\frac{\alpha+1}{2}} \operatorname{sgn}(s)-k s+\gamma$,

$\dot{\gamma}=-n|s|^{\alpha} \operatorname{sgn}(s)-p s$,

and the equivalent control for the supertwisting control STW is

$u_{s}=-k_{1}|s|^{\frac{1}{2}} \operatorname{sgn}(s)+\gamma$,

$\dot{\gamma}=-k_{2} \operatorname{sgn}(s)$. 
Table 1 Parameter values for the SOSMC and STW controllers

\begin{tabular}{ll}
\hline Method & Parameter values \\
\hline SOSMC & $h=2, k=0.1, \alpha=0.3,0.8$ Eq. (50) \\
STW & $k_{1}=2, k_{2}=0.1$. Eq. (51) \\
\hline
\end{tabular}

It can be seen that the main differences between the two controllers are that the STW has a fixed fractional power of $1 / 2$, whereas the SOSMC has a variable fractional power $\alpha$ and it also includes linear terms $k s$ and $p$ s, i.e., the STW is a special case of the SOSMC and corresponds to a choice of $\alpha=0, k=0, p=0$ in the SOSMC.

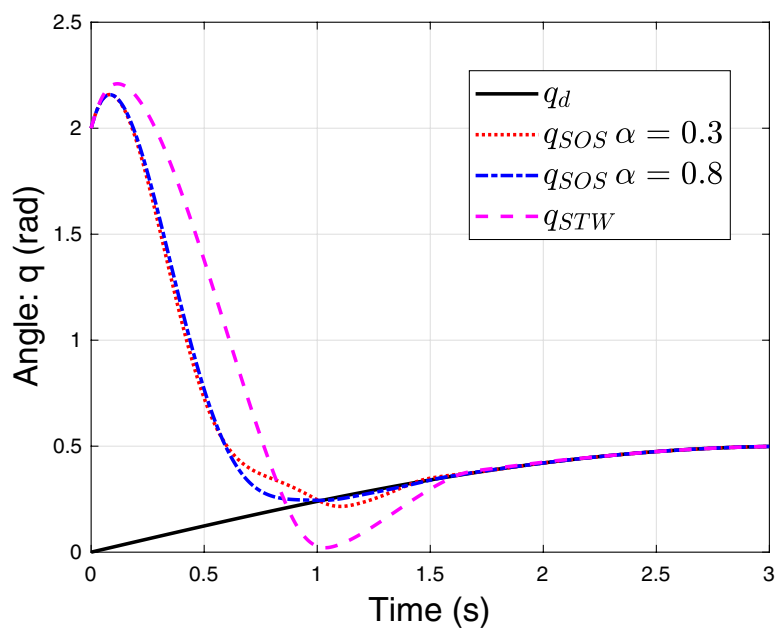

(a) Oscillation angle
In the numerical simulations, we chose the parameter values $m=1 \mathrm{~kg}, R=1-0.1 \sin (5 t) \mathrm{m}$ and the initial values for $q(0)=0.3 \mathrm{rad}, v(0)=0.5 \mathrm{rad} \cdot \mathrm{s}^{-1}$, $s(0)=10 \mathrm{rad} \cdot \mathrm{s}^{-1}$ and $\gamma(0)=0$. We also defined the error state $e$ and $\varepsilon$ as $e=q-q_{d}$ rad and $\varepsilon=v-v_{d} \mathrm{rad} \cdot \mathrm{s}^{-1}$, where the desired trajectory is $q_{d}=0.5 \sin (0.5 t) \mathrm{rad}$ and $v_{d}=0.25 \cos (0.5 t) \mathrm{rad} \cdot \mathrm{s}^{-1}$. We also assumed that the distance $R(t)=1-0.1 \sin (5 t(k))$ and $\dot{R}(t)=-0.5 \cos (5 t(k))$.

For both the SOSMC and STW control, the parameter values assumed for the control $u$ in Eq. (49) were

$\rho_{1}=0.4, \quad \rho_{2}=0.5714, \quad a=25, \quad b=8.66$,

Comparison of oscillation angle and velocity of oscillation angle for SOSM

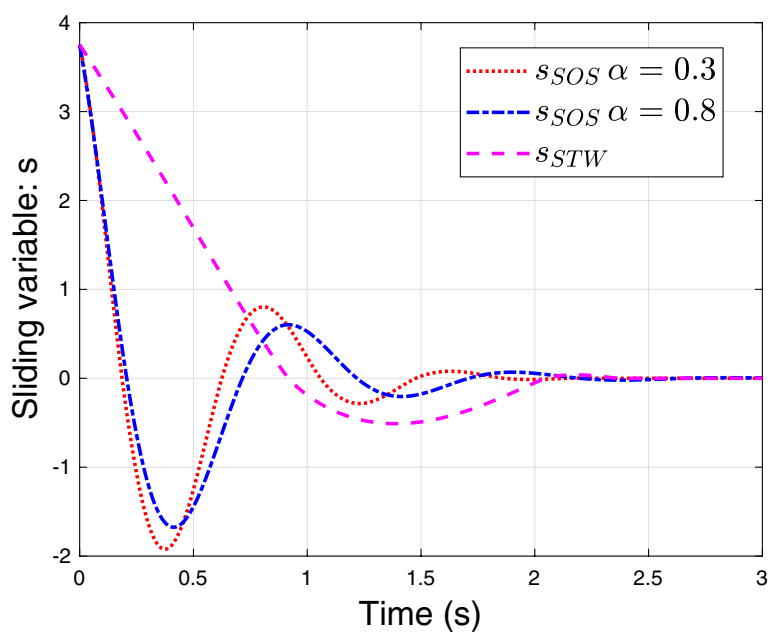

(a) Sliding variable

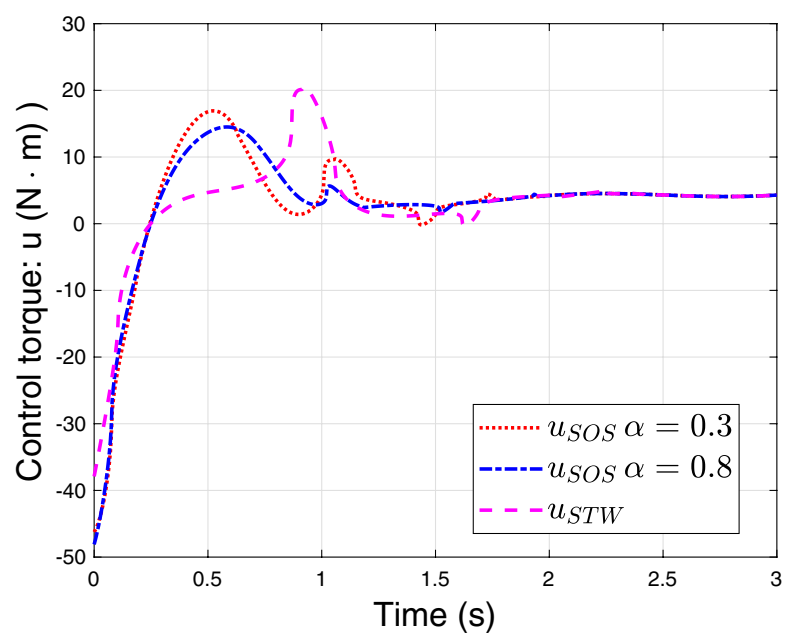

(b) Control torque

Fig. 3 Comparison of sliding variables and torques for SOSMC and STW 
and the parameter values assumed for the control $u_{s}$ in Eqs. (50) and (51) are shown in Table 1 for the SOSMC and STW.

The values for $a, b, \rho_{1}$ and $\rho_{2}$ were chosen to satisfy the conditions in Theorem 2 and the values for $h, k$, and $\alpha \in(0,1)$ were selected to have reasonable values.

Finally, the values for $n$ and $p$ in Eq. (50) were defined as

$n=1.1 h^{2} \frac{(\alpha+1)^{2}}{4}, \quad p=1.1 \frac{h^{2} k^{2}(\alpha+3)^{2}}{4 n-h^{2}(\alpha+1)^{2}}$

to satisfy the conditions (19) in Theorem 3.

Comparisons of the results from the smooth SOSMC for the values of $\alpha=0.3,0.8$ and for the STW are shown in Figs. 2 and 3. As noted above, the STW corresponds to a choice of $\alpha=0, k=0, p=0$ in the SOSMC. It can be seen that both the smooth SOSMC and the STW give fast finitetime convergence to the desired states with the order of convergence being SOSMC with $\alpha=0.8$, SOSMC with $\alpha=0.3$ and STW. In addition, the STW has a larger overshoot in oscillation angle than the SOSMC.

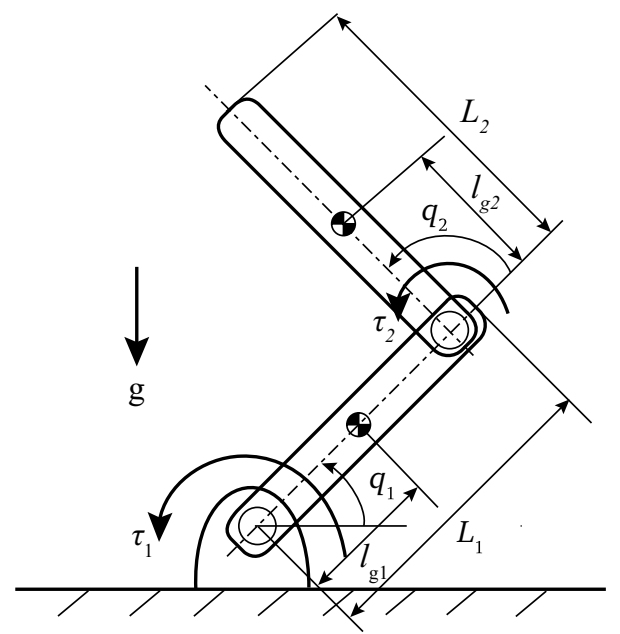

\subsection{Two-link robotic manipulator}

We have carried out numerical simulations for the multivariable system of the two-link robotic manipulator shown in Fig. 4.

From [41], the Lagrangian equations of motion for the manipulator can be written in the form of (7) as

$M(q) \ddot{q}+C(q, \dot{q}) \dot{q}+g(q)+D(t, q, \dot{q})=\tau$,

where $q=\left[\begin{array}{ll}q_{1} & q_{2}\end{array}\right]^{T}$ denotes the vector of joint positions $(\mathrm{rad}), \dot{q}=\left[\begin{array}{ll}\dot{q}_{1} & \dot{q}_{2}\end{array}\right]^{T}$ is the vector of joint velocities $\left(\mathrm{rad} \cdot \mathrm{s}^{-1}\right)$, $\tau=\left[\begin{array}{ll}\tau_{1} & \tau_{2}\end{array}\right]^{T}$ is the vector of control torques $(N \cdot m), M(q)$ is the inertia matrix $\left(\mathrm{kg} \cdot \mathrm{m}^{2}\right), C(q, \dot{q})$ is the centrifugal-Coriolis matrix $\left(\mathrm{kg} \cdot \mathrm{m}^{2} \cdot \mathrm{s}^{-1}\right), g(q)$ is the gravitational force vector $(N \cdot m)$ and $D(t, q, \dot{q})$ is the disturbance vector $(N \cdot m)$.

The meanings of the physical parameters of the manipulator and the values used in the simulations are summarized in Table 2.

The inertia matrix for the manipulator in (54) is

$M(q)=\left[\begin{array}{ll}m_{11} & m_{12} \\ m_{21} & m_{22}\end{array}\right]$,

where

$m_{11}=m_{1} l_{g 1}^{2}+m_{2} l_{g 2}^{2}+m_{2} L_{1}^{2}+2 m_{2} L_{1} l_{g 2} \cos \left(q_{2}\right)+J_{1}+J_{2}$,

$m_{12}=m_{21}=m_{2} l_{g 2}^{2}+m_{2} L_{1} l_{g 2} \cos \left(q_{2}\right)+J_{2}$,

$m_{22}=m_{2} I_{g 2}^{2}+J_{2}$.

The centrifugal-Coriolis matrix is

$C(q, \dot{q})=\left[\begin{array}{ll}c_{11} & c_{12} \\ c_{21} & c_{22}\end{array}\right]$,

where

Fig. 4 The two-link robotic manipulator [41]

Table 2 Physical parameters for two-link robotic manipulator [41]

\begin{tabular}{llll}
\hline Parameter & Definition & Units & Value \\
\hline$m_{1}$ & Mass of 1st link & $\mathrm{kg}$ & 1 \\
$m_{2}$ & Mass of 2nd link & $\mathrm{kg}$ & 1 \\
$I_{g 1}$ & Distance from 1st joint to center of mass of 1st link & $\mathrm{m}$ & 0.5 \\
$I_{g 2}$ & Distance from 2nd joint to center of mass of 2nd link & $\mathrm{m}$ & 1 \\
$L_{1}$ & Length of 1st link & $\mathrm{m}$ & 1 \\
$L_{2}$ & Length of 2nd link & $\mathrm{m}$ & 2 \\
$J_{1}$ & Moment of inertia of 1st link about its centroid & $\mathrm{kg} \cdot \mathrm{m}^{2}$ & 0.0833 \\
$J_{2}$ & Moment of inertia of 2nd link about its centroid & $\mathrm{kg} \cdot \mathrm{m}^{2}$ & 0.33 \\
$g$ & Gravitational acceleration & $\mathrm{m} \cdot \mathrm{s}^{-2}$ & 9.81 \\
\hline
\end{tabular}


$c_{11}=-m_{2} L_{1} l_{g 2} \sin \left(q_{2}\right) \dot{q}_{2}$,

$c_{12}=-m_{2} L_{1} l_{g 2} \sin \left(q_{2}\right)\left(\dot{q}_{1}+\dot{q}_{2}\right)$,

$c_{21}=m_{2} L_{1} I_{g 2} \sin \left(q_{2}\right) \dot{q}_{1}$,

$c_{22}=0$.

The gravitational force vector is

$g(q)=\left[\begin{array}{l}g_{1} \\ g_{2}\end{array}\right]$

where

$g_{1}=\left(m_{1} I_{g 1}+m_{2} L_{1}\right) g \sin \left(q_{1}\right)+m_{2} l_{g 2} g \sin \left(q_{1}+q_{2}\right)$

$g_{2}=m_{2} l_{g 2} g \sin \left(q_{1}+q_{2}\right)$.

The disturbance vector is defined as

$D(t, q, \dot{q}))=\left[\begin{array}{l}D_{1} \\ D_{2}\end{array}\right]$,

where $D_{1}$ and $D_{2}$ are assumed to satisfy Assumption 1 .

The system (54) can be written in the form of (8) as

$\dot{q}_{1}=v_{1}, \quad \dot{v}_{1}=\ddot{q}_{1}=u_{1}+f_{1}+d_{1}$,

$\dot{q}_{2}=v_{2}, \quad \dot{v}_{2}=\ddot{q}_{2}=u_{2}+f_{2}+d_{2}$,

where

$u_{1}=\frac{1}{\operatorname{det}(M)}\left(m_{22} \tau_{1}-m_{12} \tau_{2}\right)$,

$u_{2}=\frac{1}{\operatorname{det}(M)}\left(-m_{21} \tau_{1}+m_{11} \tau_{2}\right)$,

$f_{1}=\frac{1}{\operatorname{det}(M)}\left(m_{22}\left(-\left(c_{11} v_{1}+c_{12} v_{2}\right)-g_{1}\right)-m_{12}\left(-c_{21} v_{1}-g_{2}\right)\right)$,

$f_{2}=\frac{1}{\operatorname{det}(M)}\left(-m_{21}\left(-\left(c_{11} v_{1}+c_{12} v_{2}\right)-g_{1}\right)+m_{11}\left(-c_{21} v_{1}-g_{2}\right)\right)$,

$d_{1}=\frac{1}{\operatorname{det}(M)}\left(-m_{22} D_{1}+m_{12} D_{2}\right)$,

$d_{2}=\frac{1}{\operatorname{det}(M)}\left(m_{21} D_{1}-m_{11} D_{2}\right)$.

From Eqs. (10), (12) and (17), we can explicitly write the control law as $s_{1}=\varepsilon_{1}+\int_{0}^{t}\left(a_{1}\left|e_{1}\right|^{\rho_{1}} \operatorname{sgn}\left(e_{1}\right)+b_{1}\left|\varepsilon_{1}\right|^{\rho_{2}} \operatorname{sgn}\left(\varepsilon_{1}\right)\right) d \tau$,

$s_{2}=\varepsilon_{2}+\int_{0}^{t}\left(a_{2}\left|e_{2}\right|^{\rho_{1}} \operatorname{sgn}\left(e_{2}\right)+b_{2}\left|\varepsilon_{2}\right|^{\rho_{2}} \operatorname{sgn}\left(\varepsilon_{2}\right)\right) d \tau$,

and

$f_{e 1}=f_{1}-\ddot{q}_{d 1}=f 1-\dot{v}_{d 1}, \quad f_{e 2}=f_{2}-\ddot{q}_{d 2}=f_{2}-\dot{v}_{d 2}$.

By using (60), we obtained the control torques as

$\tau_{1}=m_{11} u_{1}+m_{12} u_{2}, \quad \tau_{2}=m_{21} u_{1}+m_{22} u_{2}$,

where $u_{1}$ and $u_{2}$ are given in (61).

\subsection{Numerical results for the two-link robot manipulator}

We assumed that the desired trajectories $q_{d 1}(t), q_{d 2}(t)$ and the disturbances $D_{1}, D_{2}$ were given by

$q_{d 1}=1+0.4 \sin (0.5 \pi t) \mathrm{rad}, \quad q_{d 2}=1-0.2 \sin (0.8 \pi t) \mathrm{rad}$, $D_{1}=D_{2}=0.01 \sin (t) N \cdot m$.

The initial conditions of the manipulator were set as $q_{1}(0)=q_{2}(0)=0 \mathrm{rad}$ and $v_{1}(0)=v_{2}(0)=0 \mathrm{rad} \cdot \mathrm{s}^{-1}$. The initial conditions for the sliding vector components were assumed to be $s_{1}(0)=10$ and $s_{2}(0)=-10$. It was also assumed that the initial conditions for $\gamma_{1}(t)$ and $\gamma_{2}(t)$ were $\gamma_{1}(0)=\gamma_{2}(0)=0$.

The values assumed for the parameters in the control law (61) are summarized in Table 3. The values for $a_{1}=a_{2}$ $b_{1}=b_{2}$ and $\rho_{1}$ were chosen to satisfy the conditions in Theorem 2 and the values for $h_{1}=h_{2}, k_{1}=k_{2}$, and $\alpha \in(0,1)$ were selected to have reasonable values. Finally, the values for $n_{1}=n_{2}$ and $p_{1}=p_{2}$ were defined as

$$
n_{1}=1.1 h_{1}^{2} \frac{(\alpha+1)^{2}}{4}, \quad p_{1}=1.1 \frac{h_{1}^{2} k_{1}^{2}(\alpha+3)^{2}}{4 n_{1}-h_{1}^{2}(\alpha+1)^{2}}
$$

to satisfy the conditions (19) in Theorem 3.

$$
\begin{aligned}
& u_{1}=f_{e 1}-a_{1}\left|e_{1}\right|^{\rho_{1}} \operatorname{sgn}\left(e_{1}\right)-b_{1}\left|\varepsilon_{1}\right|^{\rho_{2}} \operatorname{sgn}\left(\varepsilon_{1}\right)-h_{1}\left|s_{1}\right|^{\frac{\alpha+1}{2}} \operatorname{sgn}\left(s_{1}\right)-k_{1} s_{1}+\gamma_{1}, \\
& \dot{\gamma_{1}}=-n_{1}\left|s_{1}\right|^{\alpha} \operatorname{sgn}\left(s_{1}\right)-p_{1} s_{1}, \\
& u_{2}=f_{e 2}-a_{2}\left|e_{2}\right|^{\rho_{1}} \operatorname{sgn}\left(e_{2}\right)-b_{2}\left|\varepsilon_{2}\right|^{\rho_{2}} \operatorname{sgn}\left(\varepsilon_{2}\right)-h_{2}\left|s_{2}\right|^{\frac{\alpha+1}{2}} \operatorname{sgn}\left(s_{2}\right)-k_{2} s_{2}+\gamma_{2}, \\
& \dot{\gamma_{2}}=-n_{2}\left|s_{2}\right|^{\alpha} \operatorname{sgn}\left(s_{2}\right)-p_{2} s_{2},
\end{aligned}
$$

where the errors, the sliding surfaces and the $f_{e}$ are defined respectively by

$e_{1}=q_{1}-q_{d 1}, \quad \varepsilon_{1}=v_{1}-v_{d 1}, \quad e_{2}=q_{2}-q_{d 2}, \quad \varepsilon_{2}=v_{2}-v_{d 2}$,
Table 3 Parameter values for the control law (61)

\begin{tabular}{llllll}
\hline$\rho_{1}$ & $a_{1}=a_{2}$ & $b_{1}=b_{2}$ & $\alpha$ & $h_{1}=h_{2}$ & $k_{1}=k_{2}$ \\
\hline 0.4 & 25 & 8.66 & 0.4 & 2 & 0.1
\end{tabular}


Table 4 Eigenvalues of $\Pi, Q$ and $R$

\begin{tabular}{lllr}
\hline Matrix & $\lambda_{1}$ & $\lambda_{2}$ & \multicolumn{1}{c}{$\lambda_{3}$} \\
\hline$\Pi$ & 0.6457 & 0.7737 & 5.3145 \\
$Q$ & 0.5615 & 1.3516 & 10.7505 \\
$R$ & 0.0001 & 0.0673 & 1.2741 \\
\hline
\end{tabular}

Substituting the parameter values in Table 3 into Eqs. (26), (34) and (35), we obtain

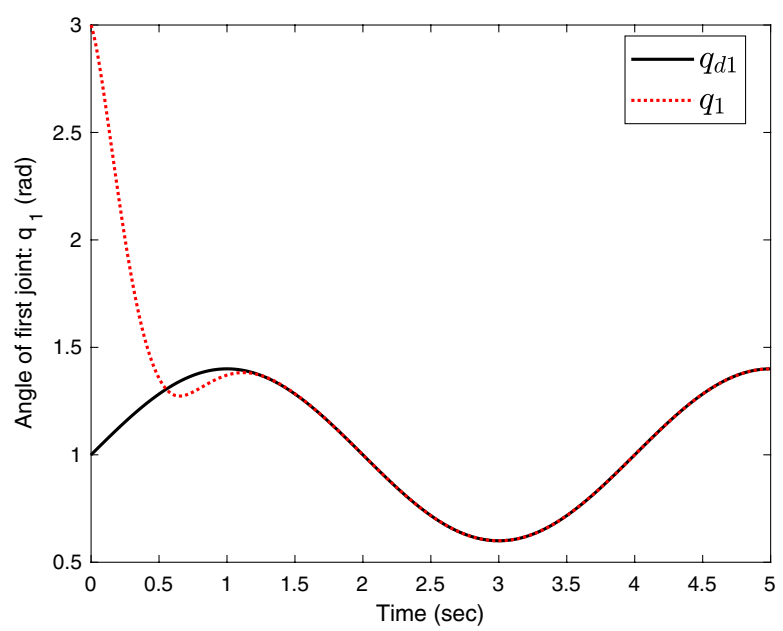

(a) First joint

$$
\begin{aligned}
& \Pi_{1}=\Pi_{2}=\Pi=\left[\begin{array}{ccc}
5.0800 & 0.1000 & -1.0000 \\
0.1000 & 0.6538 & -0.0500 \\
-1.0000 & -0.0500 & 1.0000
\end{array}\right], \\
& Q_{1}=Q_{2}=Q=\left[\begin{array}{ccc}
9.9120 & 0 & -2.8000 \\
0 & 1.3516 & 0 \\
-2.8000 & 0 & 1.4000
\end{array}\right],
\end{aligned}
$$

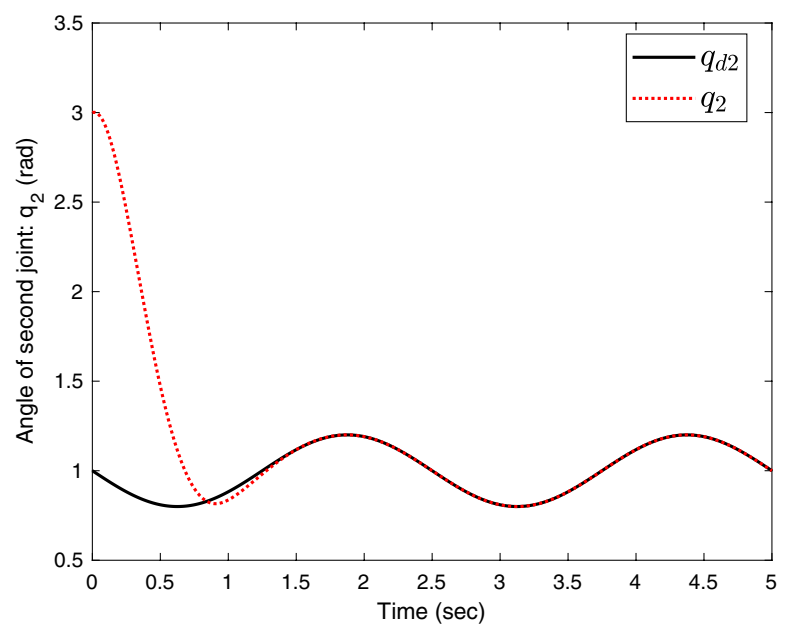

(b) Second joint

Fig. 5 Plots of positions $q_{d 1}, q_{1}$ of first joint and $q_{d 2}, q_{2}$ of second joint (SOSMC)

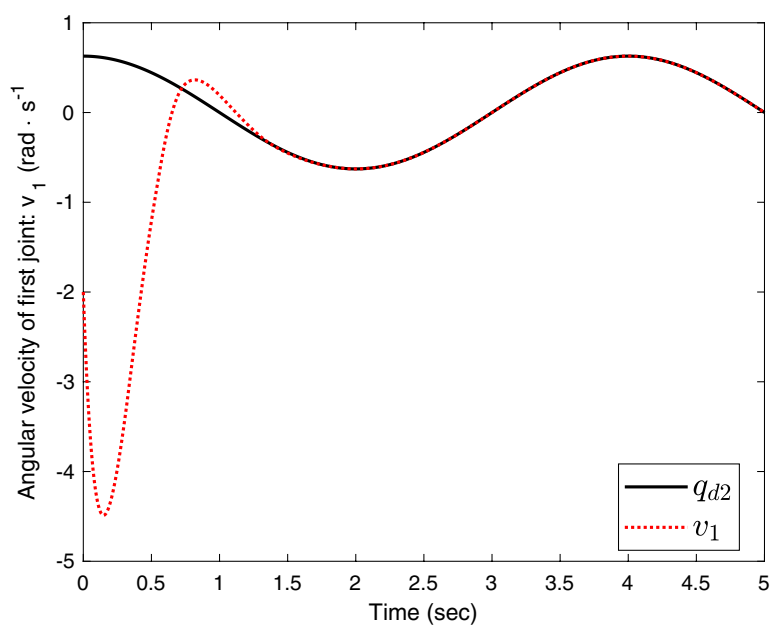

(a) First joint

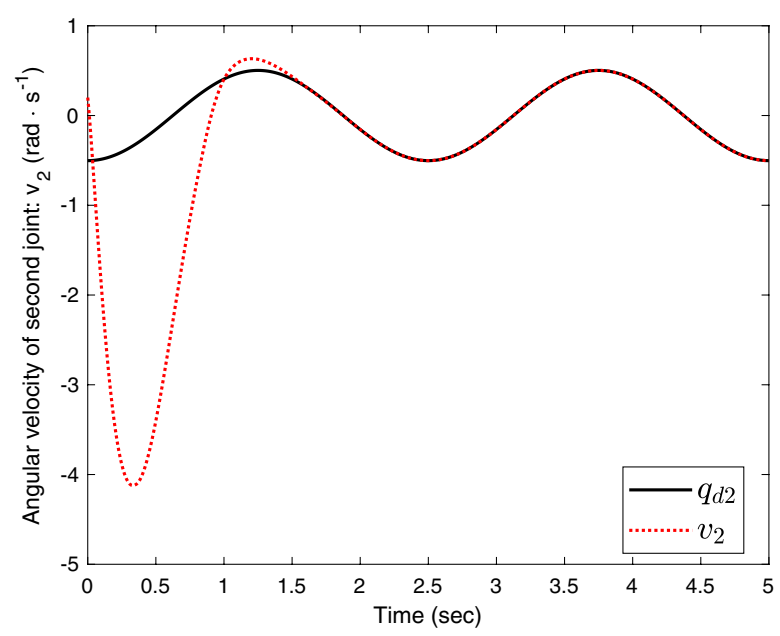

(b) Second joint

Fig. 6 Plots of velocities $\dot{q}_{d 1}, v_{1}$ of first joint and $\dot{q}_{d 2}, v_{2}$ of second joint (SOSMC) 


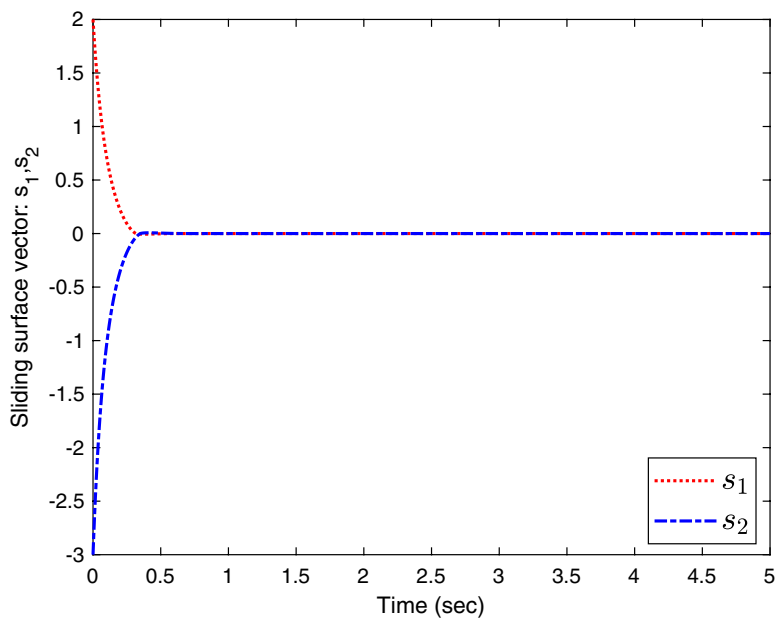

(a) Sliding surface

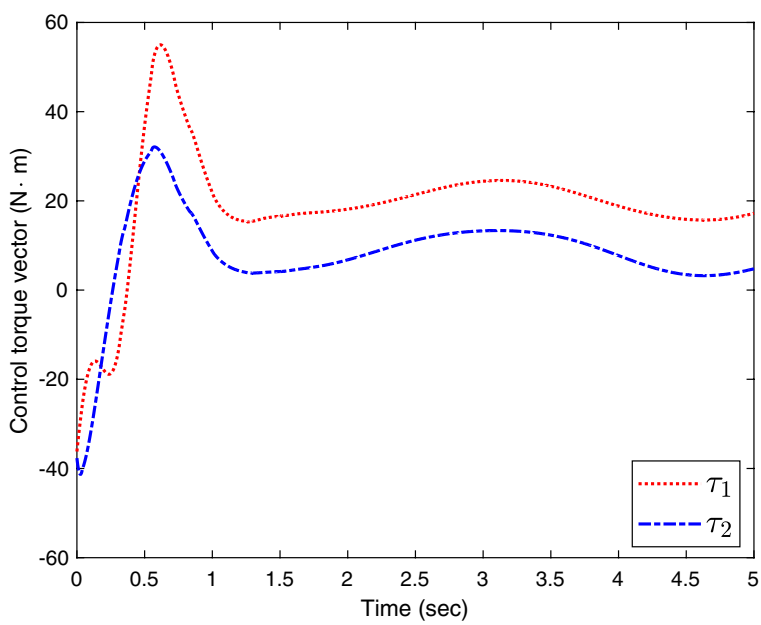

(b) Control torque

Fig. 7 Plots of sliding surface vectors $s_{1}, s_{2}$ and external torques $\tau_{1}, \tau_{2}$ (SOSMC)

Table 5 Parameter values of STW and STL for comparison with SOSMC

\begin{tabular}{ll}
\hline Method & Parameter values \\
\hline STW & $k_{11}=k_{12}=2, k_{21}=k_{22}=n_{1}$ (Eq. 67) \\
STL & $k_{11}=k_{12}=2, k_{21}=k_{22}=0.1$, \\
& $k_{31}=k_{32}=n_{1}$ (Eq. 67) \\
& $b_{11}=b_{12}=0.5, b_{21}=b_{22}=1$ \\
\hline
\end{tabular}

$R_{1}=R_{2}=R=\left[\begin{array}{ccc}1.1756 & 0 & -0.3400 \\ 0 & 0.0659 & -0.0100 \\ -0.3400 & -0.0100 & 0.1000\end{array}\right]$.

As shown in Table 4, the eigenvalues of $\Pi, Q$ and $R$ are all positive, confirming that $\Pi, Q$ and $R$ are positive-definite matrices.

Plots of the results obtained from the numerical simulations are shown in Figs. 5, 6 and 7. Figure 5a and b show the trajectories of $q_{1}$ and $q_{2}$ and the desired trajectories $q_{d 1}$ and $q_{d 2}$ and Fig. $6 a$ and $b$ show the velocities and the desired velocities of the two joints. It can be seen that the controller forces the trajectories into a neighborhood of the desired trajectories in less than $2 \mathrm{~s}$. The time histories of the control torques are shown in Fig. 7a. It can be seen that the controls rapidly converge to an oscillatory behavior which keeps the actual trajectories of $q_{1}$ and $q_{2}$ close to the desired trajectories $q_{d 1}$ and $q_{d 2}$. The convergence of the sliding surface variables $s_{1}$ and $s_{2}$ are shown in Fig. $7 \mathrm{~b}$. It can be seen that the variables initially oscillate and then converge to a neighborhood of zero, i.e., to a neighborhood of the sliding surface, in approximately $4 \mathrm{~s}$.

\subsection{Comparison of SOSMC with super-twisting (STW) and super-twisting like (STL) algorithms for two-link robot manipulator}

In this section, we compare the performance of our smooth SOSMC algorithm with the performance of the STW algorithm of [16] and the STL algorithm of [17]. In the comparisons, we will assume that the sliding mode Eq. (12) and the sliding mode controller $u_{0}$ in (14) are the same in the SOSMC, STW and STL comparisons and compare the effects of changing the smooth SOSMC controller $u_{s}$ in (15) to the equivalent STW and STL controllers. In the STW of [16], the equivalent control vector components $\left(u_{s 1}, u_{s 2}\right)$ are

$u_{s 1}=-k_{11}\left|s_{1}\right|^{1 / 2} \operatorname{sgn}\left(s_{1}\right)+\gamma_{1}, \quad \dot{\gamma}_{1}=-k_{21} \operatorname{sgn}\left(s_{1}\right)$,

$u_{s 2}=-k_{12}\left|s_{2}\right|^{1 / 2} \operatorname{sgn}\left(s_{2}\right)+\gamma_{2}, \quad \dot{\gamma}_{2}=-k_{22} \operatorname{sgn}\left(s_{2}\right)$,

and in the STL of [17], the corresponding control vector components $\left(u_{s 1}, u_{s 2}\right)$ are

$u_{s 1}=-k_{11}\left|s_{1}\right|^{b_{11}} \operatorname{sgn}\left(s_{1}\right)-k_{21}\left|s_{1}\right|^{b_{21}} \operatorname{sgn}\left(s_{1}\right)+\gamma_{1}$,
$\dot{\gamma}_{1}=-k_{31} \operatorname{sgn}\left(s_{1}\right)$
$u_{s 2}=-k_{12}\left|s_{2}\right|^{b_{12}} \operatorname{sgn}\left(s_{2}\right)-k_{22}\left|s_{2}\right|^{b_{22}} \operatorname{sgn}\left(s_{2}\right)+\gamma_{2}$
$\dot{\gamma}_{2}=-k_{32} \operatorname{sgn}\left(s_{2}\right)$.

The values of the parameters that we have used for the STW and STL controls are shown in Table 5.

We have found that the numerical simulations for STW and STL also show rapid finite-time convergence to neighborhoods of the desired trajectories within approximately three seconds with the main differences between the three methods occurring in the initial period of $3 \mathrm{~s}$. 


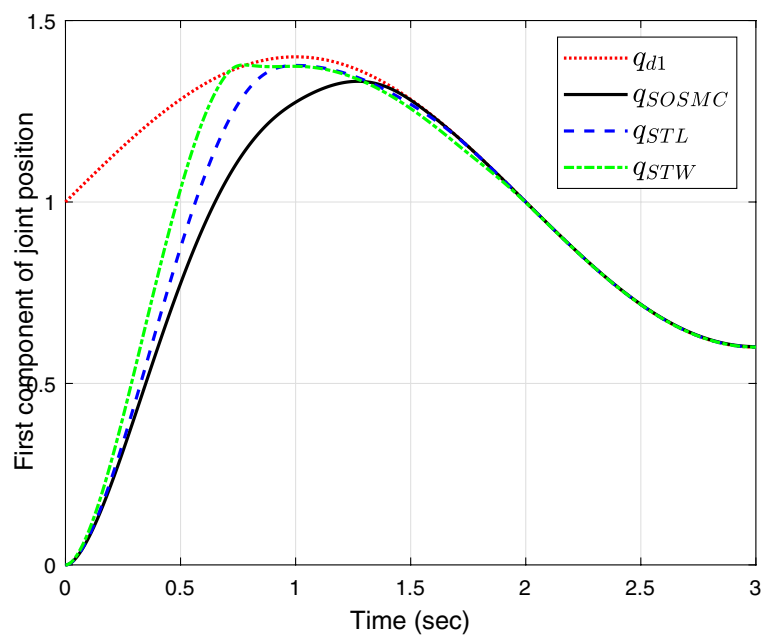

(a) First joint

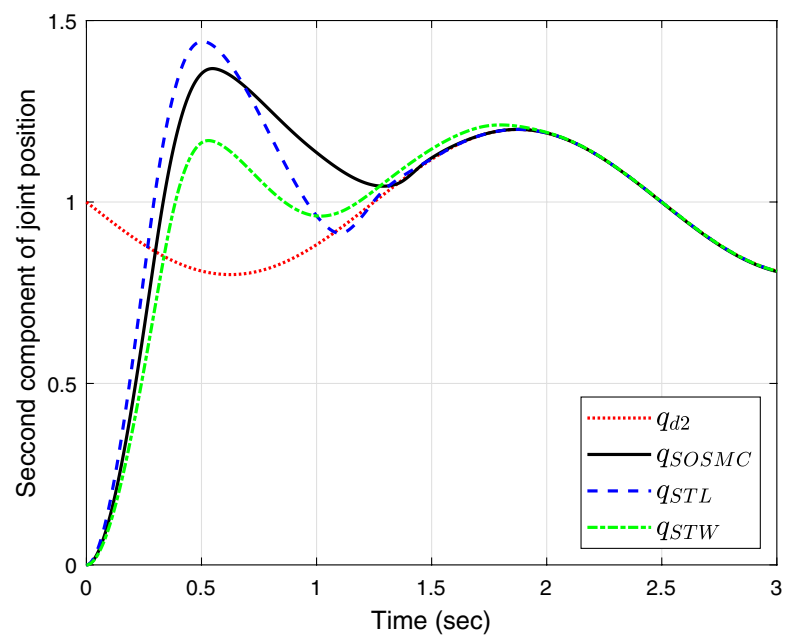

(b) Second joint

Fig. 8 Comparison of positions $q_{1}$ and $q_{2}$ of first and second joints for SOSMC, STW and STL

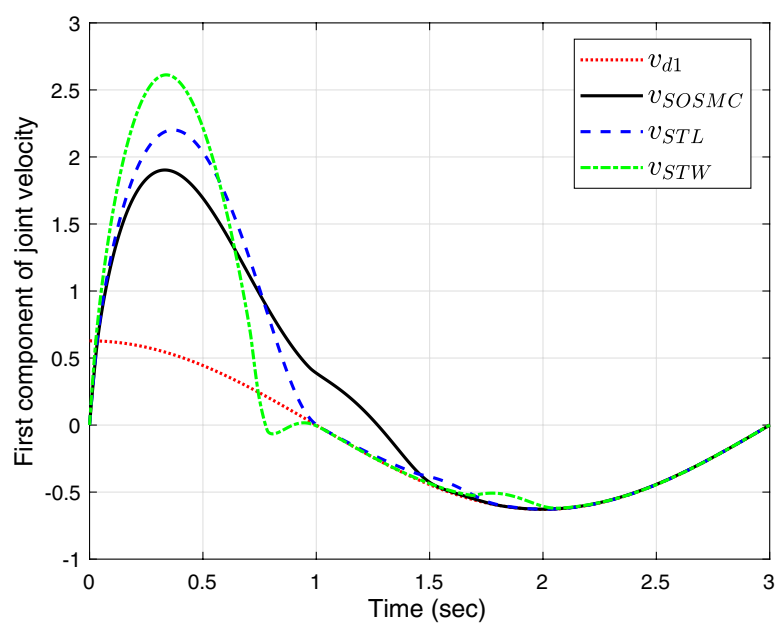

(a) First joint

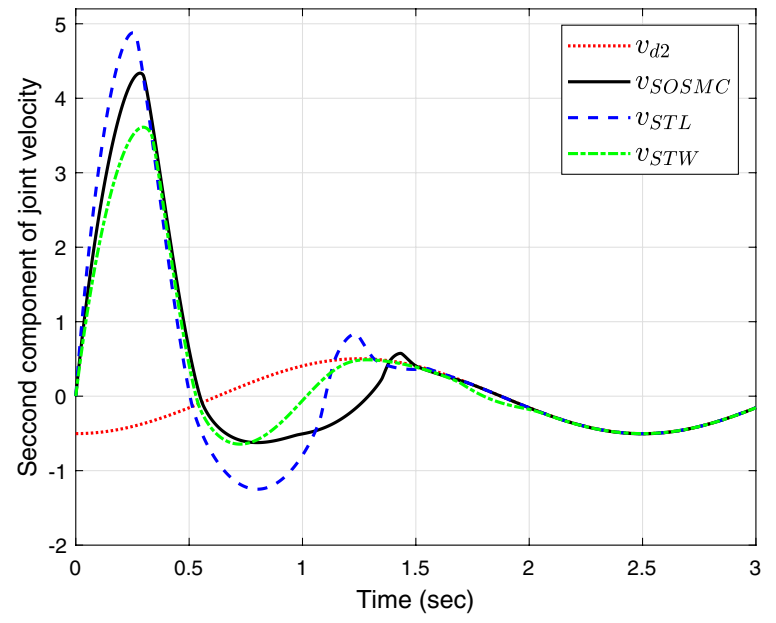

(b) Second joint

Fig. 9 Comparison of velocitites $v_{1}=\dot{q}_{1}$ and $v_{2}=\dot{q}_{2}$ of first and second joints for SOSMC, STW and STL

Comparisons of the SOSMC results with the STW and STL results are shown for the first $3 \mathrm{~s}$ in Figs. 8, 9, 10 and 11. It can be seen that the trajectories for the three algorithms all converge to the desired trajectories in less than $2 \mathrm{~s}$. Although not shown in this paper, the graphs for the period from 3 to $20 \mathrm{~s}$ for the STW and STL algorithms are the same as the graphs for SOSMC in Figs. 5, 6 and 7. A more detailed picture of the behavior of the torques in the convergence region are shown for the three algorithms in Fig. 12. It can be seen that the torque for STL algorithm shows a much higher level of chattering than the torque for the STW algorithm and that the torque for the SOSMC algorithm shows virtually no chattering.

\section{Discussion}

Because of their practical importance, many methods have been developed to control electrical and mechanical systems with uncertain parameters and external disturbances. These methods include nonlinear adaptive control [49], model predictive control [50], adaptive backstepping control [51] and, of course, sliding mode control. 


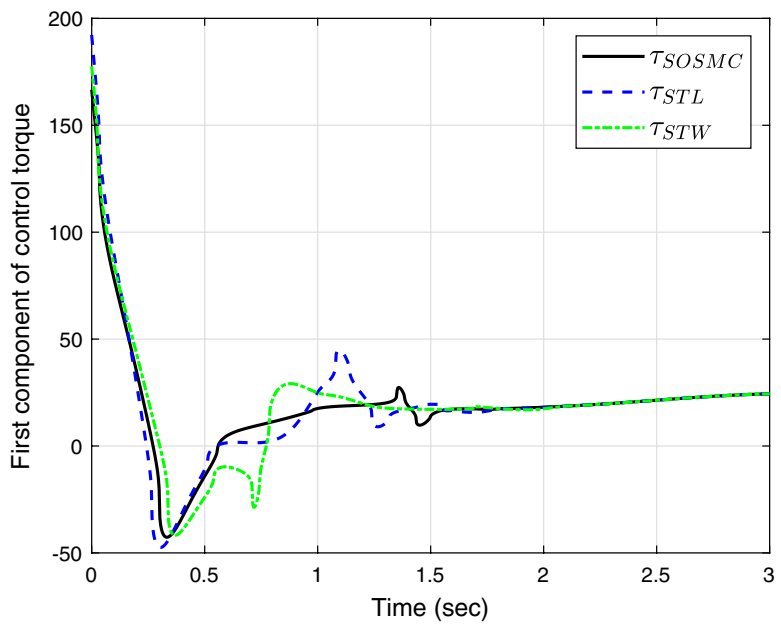

(a) $\tau_{1}$

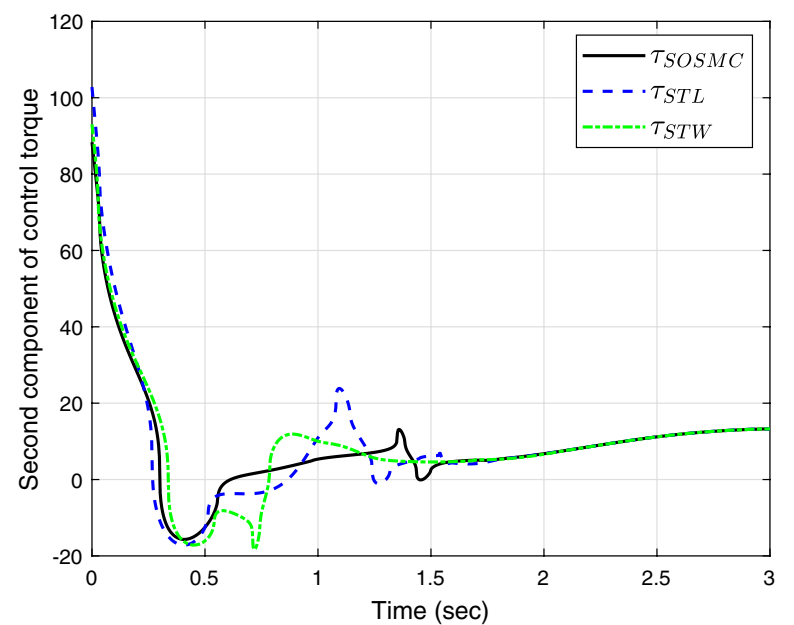

(b) $\tau_{2}$

Fig. 10 Comparison of external torques $\tau_{1}$ and $\tau_{2}$ for SOSMC, STW and STL

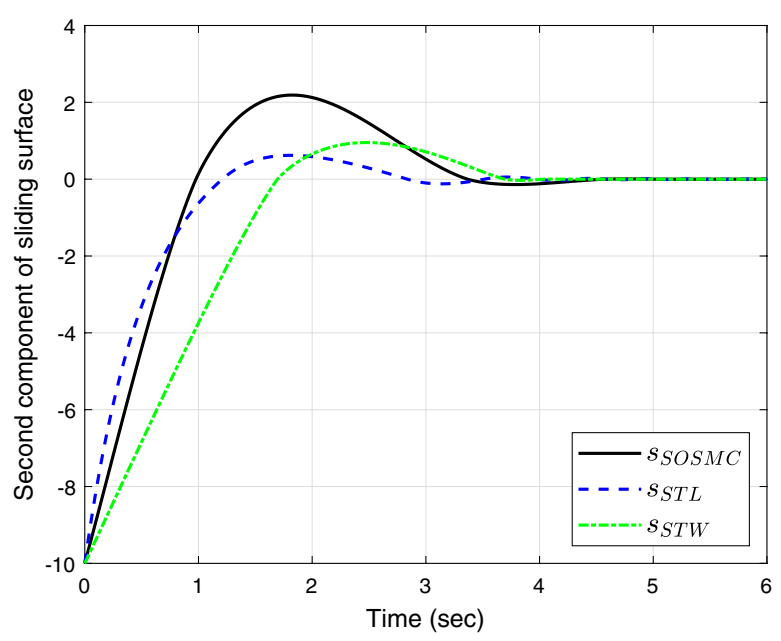

(a) $s_{1}$

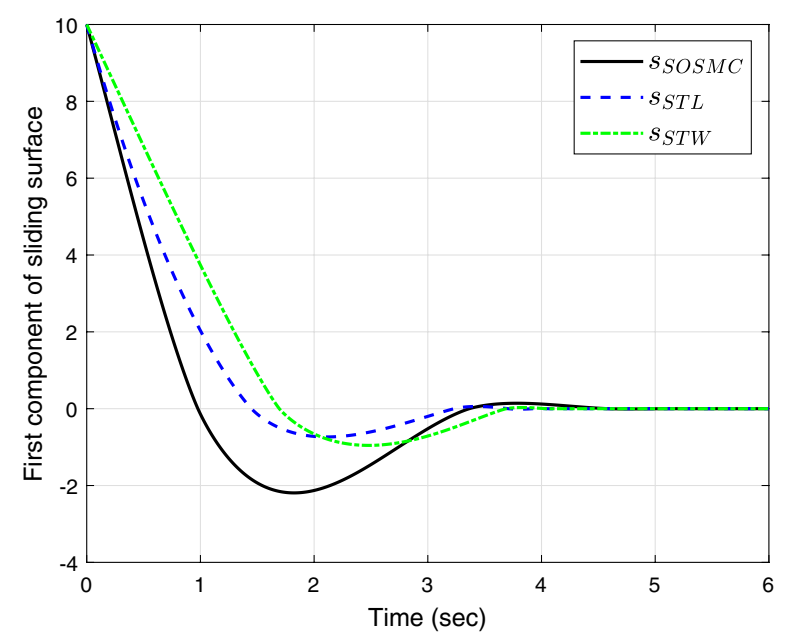

(b) $s_{2}$

Fig. 11 Comparison of sliding surface variables $s_{1}$ and $s_{2}$ for SOSMC, STW and STL

In general, as explained by Boutalbi et al. [52], the control methods can be divided into two main classes, namely, adaptive control and robust control. In adaptive control, one of the main aims is to design the controller to estimate the uncertain system parameters of the dynamical system and then to follow some specified desired dynamics. In robust control, which includes sliding mode control, the aim is to develop a simple fixed controller that yields acceptable performance for bounded disturbances.

Because of the importance of robotic manipulators, many papers have been published in recent years giving the application of various control methods to the control of these systems. These methods include proportional-derivative control [53], adaptive control [52], and integral-type saturated sliding mode control [54]. The main features of these papers are as follows.

The controller of Cruz-Zavala et al. [53] is a finite-time nonlinear scheme that includes a proportional-derivative nonlinear feedback and a feed-forward compensation term. The stability of the robot manipulators was proved for three cases (i) Finite-time trajectory-tracking without model uncertainties and perturbations, (ii) finite-time trajectory-tracking with uncertainties and without perturbations, and (iii) finite-time trajectory-tracking with model uncertainty and perturbations. In each case, the controller 


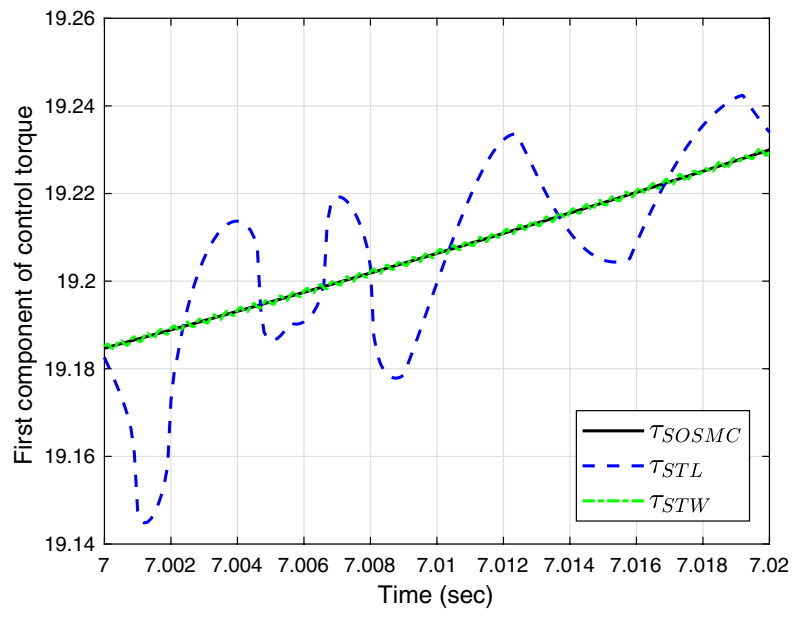

(a) STL

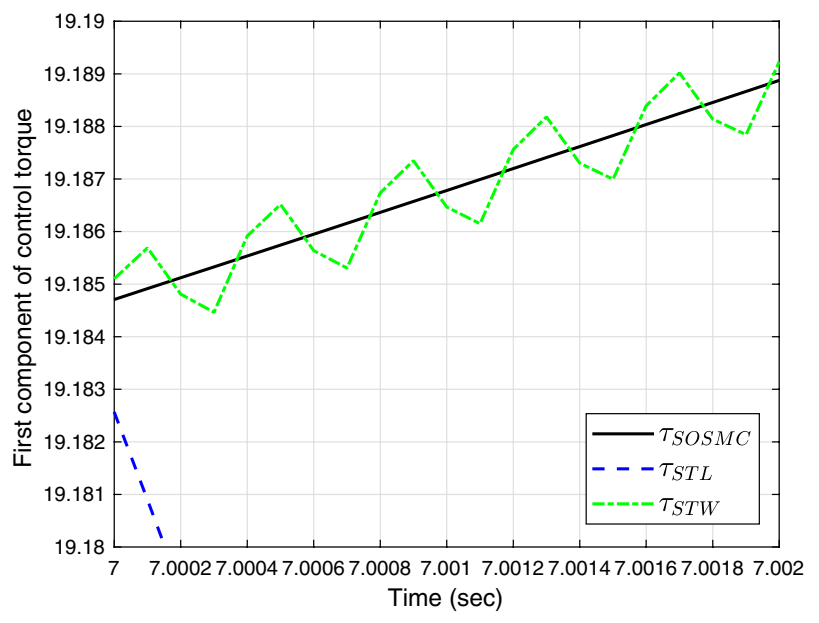

(b) STW

Fig. 12 Comparison of zoomed torques $\tau_{1}$ to show STL and STW chattering

was proved to be globally finite-time stable by using Lyapunov theory.

In the adaptive controller of Boutalbi et al. [52], the values of the parameters of the manipulator were estimated for both bounded and unbounded parameters. The finitetime stability of the controller was proved using Lyapunov theory and the weighted homogeneity principle for unknown model parameters and external disturbances.

In the integral-type saturated sliding mode controller of Guo et al. [54], the sliding surface and sliding mode controller was defined in terms of saturating functions rather than the signum functions used in our controller. They proved the reachability of the sliding surface, the finitetime convergence of the state system and the boundedness of the controller using Lyapunov-based methods.

In the present paper, we have proposed a smooth second-order sliding mode control scheme with the aim of finite-time convergence and reduced chattering for a system with bounds on the values of uncertain parameters and external disturbances and we have shown the application of the controller to the variable-length pendulum and a twolink robotic manipulator. The main differences between our paper and the papers mentioned above are that, as with sliding mode controllers, the disturbances are assumed to be bounded and no attempt is made to estimate values of uncertain parameters of the dynamical system.

\section{Conclusions}

In this paper, we have developed a smooth second-order sliding mode controller (SOSMC) for finite-time control of a class of multi-input multi-output mechanical systems with uncertain parameters and external disturbances. Our proposed sliding surface is based on an integral form and chosen such that the error states converge to the origin in finite time. Since our smooth SOSMC includes continuous first-order time derivatives of the sliding variables, the chattering that can occur with standard sliding mode controllers is reduced. By utilizing a Lyapunov-based method, we have proved that the controller drives a disturbed state of a representative mechanical system to the sliding surface in finite time and then drives the system along the sliding surface to a desired state in finite time.

Numerical simulations have been carried out for a variable-length pendulum and a two-link robotic manipulator to illustrate the effectiveness of the proposed controller. We have compared the effectiveness of the smooth SOSMC for the manipulator with the effectiveness of a super-twisting (STW) controller [16] and a super-twisting like (STL) controller [17] by setting the parameter values of our controller with the same structure as the STW and the STL controllers. The numerical results show that all three controllers give fast finite-time convergence to a neighborhood of a desired trajectory and that the SOSMC has the lowest level of chattering. As noted in subsection 4.1, the main differences between the SOSMC and STW controllers are that the STW controller has a fixed fractional power of $1 / 2$, whereas the SOSMC has a variable fractional power $\alpha$ and it also includes linear terms $k s$ and $p s$. Also, the STW and STL both include a term in Eqs. (51), (71) and (72) for $\dot{\gamma}=-k \operatorname{sgn}(s)$ which gives a discontinuity in the first derivative of the control. The equivalent SOSMC formula in Eqs. (50) and (61) is $\dot{\gamma}=-n|s|^{\alpha} \operatorname{sgn}(s)-p s$ which gives a continuous first derivative in the control. 
As shown in the simulations for the variable-length pendulum, tuning the value of $\alpha$ can improve the performance of the SOSMC. In this work, we have manually selected values of the parameters in the SOSMC. However, an optimization method can be utilized to tune them if needed for a given mechanical system, but this tuning is beyond the scope of the present paper.

Finally, the question can be asked whether our smooth sliding mode controller can be implemented in practise. Nowadays, controllers are computer based, and therefore any complicated control law can be easily implemented. Moreover, the processing unit of modern computers is very fast compared to the response of controlled systems, which means that discretized effects can be ignored. However, if the discretized effect cannot be ignored for any reason then, as in the recent work of Li et al. [55] and Wang et al. [56], the discrete-time version of the control law might need to be further investigated. We consider this as future research. In fact, our control law is not significantly more complicated than any existing SMC-based methods such as the super-twisting algorithm.

Acknowledgements The authors wish to acknowledge the valuable comments and suggestions they have received from the Managing Editor of the journal and the three reviewers. The authors would also like to acknowledge the assistance they have received from the Transfer Section of the SpringerNature editorial office in submitting this paper to SN Applied Sciences.

Author contributions All authors contributed equally to this work. Unfortunately, C.P. passed away before this paper was completed. All other authors read and approved the final manuscript.

Funding Panitnart Chawengkrittayanont would like to acknowledge financial support from a Science Achievement Scholarship of Thailand (SAST)

Data availability Data sharing is not applicable to this article as no datasets were generated or analyzed during the current study.

Code availability All computer code used is available from the corresponding author.

\section{Declarations}

Conflict of interest The authors declare that they have no conflict of interest.

Open Access This article is licensed under a Creative Commons Attribution 4.0 International License, which permits use, sharing, adaptation, distribution and reproduction in any medium or format, as long as you give appropriate credit to the original author(s) and the source, provide a link to the Creative Commons licence, and indicate if changes were made. The images or other third party material in this article are included in the article's Creative Commons licence, unless indicated otherwise in a credit line to the material. If material is not included in the article's Creative Commons licence and your intended use is not permitted by statutory regulation or exceeds the permitted use, you will need to obtain permission directly from the copyright holder. To view a copy of this licence, visit http://creativecommons. org/licenses/by/4.0/.

\section{References}

1. Utkin VI (1977) Variable structure systems with sliding modes. IEEE Trans Autom Control 22(2):212-222

2. Bartolini G, Ferrara A, Utkin VI (1995) Adaptive sliding mode control in discrete-time systems. Automatica 31(5):769-773

3. Abidi K, Jian-Xin X, Xinghuo Yu (2007) On the discrete-time integral sliding-mode control. IEEE Trans Autom Control 52(4):709-715

4. Benamor A, Chrifi-alaui L, Messaoud H, Chaabane M (2011) Sliding mode control, with integrator, for a class of MIMO nonlinear systems. Sci Res 3(5):435-444

5. Pisano A (2011) On the multi-input second-order sliding mode control of nonlinear uncertain systems. Int J Robust Nonlinear Control 22:1-14

6. Li Y, Zhang J, Wu X, Zhu Q (2014) Sliding mode control for discrete time switched systems with uncertain parameters and time delay. In: International conference on modelling, identification and control, pp 505-512. IEEE

7. Loukianov AG, Soto-Cota A, Fridman LM, Canedo JM (2004) Sliding mode control of large scale power systems, 5th Asian Control Conference (IEEE Cat. No.04EX904) 1:629-634

8. Orlov Y, Louz Y, Christofides PD (2004) Robust stabilization of infinite-dimensional systems using sliding-mode output feedback control. Int J Control 77:1115-1136

9. Feng Z, Shi $P$ (2015) Sliding mode control of singular stochastic Markov jump systems. J Latex Class Files 14:1-8

10. Song $H$, Chen S-C, Yam Y (2017) Sliding mode control for discrete-time systems with Markovian packet dropouts. IEEE Trans Cybern 47:3669-3679

11. Zhang Q, Li J (2016) Static output feedback sliding mode control for stochastic descriptor systems. In: Control and decision conference, pp 1093-1097. IEEE

12. Edwards C, Spurgeon SK (1998) Sliding mode control: theory and applications. Taylor \& Francis Ltd, London

13. Utkin VI (2013) Sliding modes in control and optimization. Communications and Control Engineering. Springer-Verlag, Berlin, Germany

14. Levant A (1993) Sliding order and sliding accuracy in sliding mode control. Int J Control 58(6):1247-1263

15. Levant A (2001) Universal single-input-single-output (SISO) sliding-mode controllers with finite-time convergence. IEEE Trans Autom Control 46(9):1447-1451

16. Levant A (2003) Higher-order sliding modes, differentiation and output-feedback control. Int J Control 76(9-10):924-941

17. Basin M, Panathula CB, Shtessel Y (2017) Multivariable continuous fixed-time second-order sliding mode control: design and convergence time estimation. IET Control Theory Appl 11:1104-1111

18. Shtessel Y, Edwards C, Fridman L, Levant A (2014) Sliding mode control and observation. Birkhauser, New York, USA

19. Utkin V (2016) Discussion aspects of high-order sliding mode control. IEEE Trans Autom Control 61(3):829-833

20. Utkin V, Poznyak A, Orlov Y, Polyakov A (2020) Conventional and high order sliding mode control. J Franklin Inst 357(15):10244-10261

21. Utkin V, Poznyak A, Orlov Y, Polyakov A (2020) Road map for sliding mode control design. Springer, Switzerland 
22. Floquet T, Barbot J-P, Perruquetti W (2003) Higher-order sliding mode stabilization for a class of nonholonomic perturbed systems. Automatica 39:1077-1083

23. Laghrouche S, Smaoui M, Plestan F, Brun X (2006) Higher order sliding mode control based on optimal approach of an electropneumatic actuator. Int J Control 79:119-131

24. Laghrouche S, Plestan F, Glumineau A (2007) Higher order sliding mode control based on integral sliding mode. Automatica 43:531-537

25. Bhat SP, Bernstein DS (2005) Geometric homogeneity with applications to finite-time stability. Math Control Signals Syst 17(2):101-127

26. Defoort M, Floquet T, Kökösy A, Perruquetti W (2009) A novel higher order sliding mode control scheme. Syst Control Lett 58(2):102-108

27. Feng $Y$, Han F, Xinghuo Yu (2014) Chattering free full-order sliding-mode control. Automatica 50(4):1310-1314

28. Shtessel YB, Shkolnikov IA, Levant A (2007) Smooth secondorder sliding modes: Missile guidance application. Automatica 43(8):1470-1476

29. Pukdeboon C (2013) Finite-time second-order sliding mode controllers for spacecraft attitude tracking. Math Probl Eng 2013:12

30. Pukdeboon C, Zinober Alan SI, Thein May-Win L (2010) Quasicontinuous higher order sliding-mode controllers for spacecraft-attitude-tracking maneuvers. IEEE Trans Indus Electron 57(4):1436-1444

31. Mondal S, Mahanta C (2013) Adaptive integral higher order sliding mode controller for uncertain systems. J Control Theory Appl 11:61-68

32. Guendouzi A, Larbi AH, Hamerlain M, Guendouzi F (2013) Higher order sliding mode control structure for a scale model autonomous helicopter. In: 3rd international conference on systems and control, pp 868-873, Algiers, Algeria

33. Meng Q, Sun Z-Y, Li Y (2018) Finite-time controller design for four-wheel-steering of electric vehicle driven by four in-wheel motors. Int J Control Autom Syst 81:52-62

34. Zhang C (2018) Finite-time convergence of an nth nonlinear system with unmatched disturbance and its application in integrated guidance and control system. Int J Model Simul Sci Comput 09(5):1850039

35. Mao J, Li S, Li Q, Yang J (2019) Design and implementation of continuous finite-time sliding mode control for 2-dof inertially stabilized platform subject to multiple disturbances. ISA Trans 84:214-224

36. Sangpet $T$, Kuntanapreeda $S$ (2020) Finite-time synchronization of hyperchaotic systems based on feedback passivation. Chaos, Solitons Fractals 132:109605

37. Wang $Z$ (2017) Adaptive smooth second-order sliding mode control method with application to missile guidance. Trans Inst Meas Control 39(6):848-860

38. Yang P, Fang Y, You-li W, Zhang D, Yang X (2018) Fast smooth second-order sliding mode control for stochastic systems with enumerable coloured noises. Int J Syst Sci 49(2):312-323

39. Moreno JA, Osorio M (2012) Strict Lyapunov functions for the super-twisting algorithm. IEEE Trans Autom Control 57(4):1035-1040
40. Shi S, Shengyuan X, Zhang B, Ma Q, Zhang Z (2019) Global second-order sliding mode control for nonlinear uncertain systems. Int J Robust Nonlinear Control 29(1):224-237

41. Bouakrif F, Zasadzinski M (2018) High order iterative learning control to solve the trajectory tracking problem for robot manipulators using Lyapunov theory. Trans Inst Meas Control 40(15):4105-4114

42. Slotine JJE, Li W (1991) Applied nonlinear control. Prentice Hall, New Jersey, USA

43. Khalil HK (2002) Nonlinear systems. Prentice Hall, New Jersey, USA

44. Luenberger DG (1979) Introduction to dynamic systems: theory, models, and applications. John Wiley \& Sons, New York, NY

45. Shuanghe Yu, Xinghuo Yu, Shirinzadeh B, Man Z (2005) Continuous finite-time control for robotic manipulators with terminal sliding mode. Automatica 41(11):1957-1964

46. Liu S, Geng Z, Sun J (2015) Finite-time attitude control: a finite-time passivity approach. IEEE/CAA J Automatica Sinica 2(1):102-108

47. Davila J, Fridman L, Levant A (2005) Second-order sliding-mode observer for mechanical systems. IEEE Trans Autom Control 50(11):1785-1789

48. Levant A (2007) Principles of 2-sliding mode design. Automatica 43:576-586

49. Astolfi A, Karagiannis D, Ortega R (2007) Nonlinear and adaptive control with applications. Communications and Control Engineering. Springer-Verlag, London, UK

50. Alba CB (2012) Model predictive control. Advanced Textbooks in Control and Signal Processing. Springer-Verlag, London

51. Zhou J, Wen C (2008) Adaptive backstepping control of uncertain systems: nonsmooth nonlinearities. Interactions or Timevariations. Lecture Notes in Control and Information Sciences. Springer-Verlag, Berlin, Germany

52. Boutalbi O, Benmahammed K, Boukezata B (2021) An adaptive finite-time stable control law for manipulator robots with unknown parameters. Int J Robust Nonlinear Control 31(11): 5218-5243

53. Cruz-Zavala E, Nuno E, Moreno JA (2021) Robust trajectorytracking in finite-time for robot manipulators using nonlinear proportional-derivative control plus feed-forward compensation. Int J Robust Nonlinear Control 31(9):3878-3907. https:// doi.org/10.1002/rnc.5334

54. Guo Y, Huang B, Li AJ, Wang CQ (2019) Integral sliding mode control for Euler-Lagrange systems with input saturation. Int J Robust Nonlinear Control 29:1088-1100. https://doi.org/10. 1002/rnc.4431

55. Li Shihua, Haibo Du, Xinghuo Yu (2014) Discrete-time terminal sliding mode control systems based on Euler's discretization. IEEE Trans Autom Control 59(2):546-552

56. Wang B, Brogliato B, Acary V, Boubakir A, Plestan F (2015) Experimental comparisons between implicit and explicit implementations of discrete-time sliding mode controllers: Toward input and output chattering suppression. IEEE Trans Control Syst Technol 21(5):2071-2075

Publisher's Note Springer Nature remains neutral with regard to jurisdictional claims in published maps and institutional affiliations. 